\title{
A Computational Model for Quantum Measurement
}

\author{
R. Srikanth* \\ Raman Research Institute, \\ Bangalore- 560 080, Karnataka, India.
}

\begin{abstract}
Is the dynamical evolution of physical systems objectively a manifestation of information processing by the universe? We find that an affirmative answer has important consequences for the measurement problem. In particular, we calculate the amount of quantum information processing involved in the evolution of physical systems, assuming a finite degree of fine-graining of Hilbert space. This assumption is shown to imply that there is a finite capacity to sustain the immense entanglement that measurement entails. When this capacity is overwhelmed, the system's unitary evolution becomes computationally unstable and the system suffers an information transition ('collapse'). Classical behaviour arises from the rapid cycles of unitary evolution and information transition. Thus, the fine-graining of Hilbert space determines the location of the 'Heisenberg cut', the mesoscopic threshold separating the microscopic, quantum system from the macroscopic, classical environment. The model can be viewed as a probablistic complement to decoherence, that completes the measurement process by turning decohered improper mixtures of states into proper mixtures. It is shown to provide a natural resolution to the measurement problem and the basis problem.
\end{abstract}

PACS numbers: 03.65.Ta

Keywords:

\section{INTRODUCTION}

Inspite of the tremendous success of standard quantum mechanics, its interpretational aspects continue to puzzle us, particularly in regard to measurement and its extrapolation to the macroscopic level [1, 2]. Although an overwhelming number of current studies and applications of quantum mechanics do not depend on the philosophical resolutions of these difficulties, they will become important for understanding future experiments where advancing technology will permit one to probe mesoscopic phenomena [3]. Further, they can help resolve the measurement problem, a subtle but arguably important problem in quantum epistemology and foundations. In simple words, it is concerned with understanding how macroscopic phenomena, in specific measurement outcomes, are classical even though the underlying microscopic states exist as quantum mechanical superpositions [4]. Let a system $S$ to be measured be in the pure state $|\psi\rangle=\sum_{i=1}^{n} a_{i}|i\rangle$, with $\sum_{i=1}^{n}\left|a_{i}\right|^{2}=1$ where $\{|i\rangle\}$ are a complete set of eigenstates of some observable $\hat{A}$ that can be measured by a measuring apparatus $M$. Formally, measurement is represented by the action of the projection operator $\pi_{i}=|i\rangle\langle i|$ on $|\psi\rangle$, with probablity $\left|a_{i}\right|^{2}$, and more generally, by the action of arbitrary measurement operators [5, 6]. Under such action, the system $S$ is said to undergo an irreversible 'reduction of the state vector', 'collapse of the wavefunction' or 'quantum leap/jump' to an eigenstate. In contrast, Schrödinger evolution is given by the deterministic action of a unitary operator. This difference in the actions of unitary evolution and measurement is the simplest manifestation of the measurement problem.

To be precise, we need to take into consideration the measuring apparatus. Following von Neumann 7], we visualize the measurement as beginning with $S$ interacting with $M$ in the 'ready' state $|R\rangle$. During this "pre-measurement" phase, the interaction Hamiltonian entangles $S$ with $M$. Assuming the apparatus can be characterized by a single degree of freedom, represented by the states $\left\{\left|\xi_{i}\right\rangle\right\}$ that span the pointer basis, one obtains the state

$$
|\psi\rangle|R\rangle=\sum_{i} a_{i}|i\rangle|R\rangle \stackrel{\hat{H}}{\longrightarrow}|\Psi\rangle=\sum_{i} a_{i}|i\rangle\left|\xi_{i}\right\rangle
$$

whereby every state $|i\rangle$ gets correlated with a definite macroscopic apparatus state $\left|\xi_{i}\right\rangle$. Eq. (1) implies a superposition of macroscopic configurations of $M$, contrary to our everyday experience of determinateness of macroscopic objects. Expressed verbally, how/when do the logical AND's (the +'s in the summation over $i$ ) in Eq. (1) becomes OR's? This is the measurement problem. As recently demonstrated in Ref. [8], such a projective operation represents a break-down

*Electronic address: srik@rri.res.in 
in the principle of linear superposition for state vectors, irrespective of the details of the measuring apparatus and the measurement process.

The oldest attempt to make sense of this dual dynamics in quantum mechanics is the Copenhagen interpretation, due mostly to Bohr and Heisenberg [1]. It was not a single interpretation, but rather a collection of somewhat different viewpoints, the main thrust being that physical systems evolve as quantum objects, whereas measuring apparatuses and their outcomes are to be represented classically: if a quantity $\mathcal{Q}$ is measured in system $S$ at time $t$ then $\mathcal{Q}$ has a particular value in $S$ at time $t$. A difficulty with the Copenhagen interpretation appears to be that it divides the physical world into the microscopic, quantum realm of physical systems, governed by the Schrödinger equation and linear superpositions, and the macrocopic, classical realm of measuring apparatuses, governed by Newtonian dynamics. However, this pragmatic and admirably minimalist approach does not clarify exactly where this "Heisenberg cut" between the quantum system and the classical measuring apparatus or observer lies, and moreover how and also why its existence does not weaken the premise that all phenomena should be describable as quantum mechanical. As a criticism of this dual unitary-and-projection dynamics, Schrödinger proposed his well known cat paradox [4, 9].

To account for measurements, since $M$ in Eq. (11) does not admit a classical description, von Neumann 7] introduces a second apparatus $M^{\prime}$ which observes and collapses $M$, and possibly a third apparatus $M^{\prime \prime}$, and so on until there is a final measurement, which has a definite result and is not described by quantum mechanics, except in the statistical sense. The Bassi-Ghirardi theorem [8] implies that such a final measurement which terminates this chain of efficient causes is inevitable. This line of argument suggests that the "cut" between quantum and classical worlds can be postponed to various depths of measurement, but not avoided.

An important role in the measurement process is played by (environment induced) decoherence [12], the practically irreversible quenching of certain off-diagonal elements from the density matrix of a bounded but open system 10, 11, 12, 13, 14, 15. It can be shown to arise naturally as a result of a system's unavoidable interaction and entanglement with its environment according to the Schrödinger equation for a global system under certain initial conditions. Given $\left\{\left|e_{i}\right\rangle\right\}$ as states of the environment $Q$ correlated with $M$ 's pointer states $\left\{\left|\xi_{i}\right\rangle\right\}$, after some time, process (10) leads to $|\Psi\rangle|0\rangle \longrightarrow \sum_{i} a_{i}|i\rangle\left|\xi_{i}\right\rangle\left|e_{i}\right\rangle$. The resulting density operator is:

$$
\rho_{S M Q}=\sum_{i, j} a_{i} a_{j}^{*}|i\rangle\left|\xi_{i}\right\rangle\left|e_{i}\right\rangle\langle j|\left\langle\xi_{j}\right|\left\langle e_{j}\right|,
$$

Detailed analysis of the problem shows that the states of $S M$ rapidly decohere, i.e., states $\left\{\left|e_{i}\right\rangle\right\}$ can be treated as if they are orthogonal [14]. Next, tracing over the (unknown) environmental degrees of freedom, and letting $\Pi_{i} \equiv\left|\xi_{i}\right\rangle\left\langle\xi_{i}\right|$ on $M$, we obtain:

$$
\begin{aligned}
\rho_{S M} \rightarrow \hat{\rho}_{S M} & =\operatorname{Tr}_{Q}\left(\rho_{S M Q}\right)=\sum_{i}\left|a_{i}\right|^{2}|i\rangle\left\langle i|\otimes| \xi_{i}\right\rangle\left\langle\xi_{i}\right| \\
& =\sum_{i} \Pi_{i} \rho_{S M} \Pi_{i} .
\end{aligned}
$$

Thus, the result (3b) of a non-selective (projective) measurement on $\rho_{S M}$, is the same as the reduced density operator in Eq. (3a). Hence, decoherence indeed resolves the measurement problem vis-a-vis non-selective measurements.

We note that Eq. (3) conflates two different meanings of the reduced density operator: on the one hand, the apparent ensemble (3a) resulting from tracing over $\left\{\left|e_{i}\right\rangle\right\}$, and on the other, a genuine classical mixture of pure states in (3b). Following d'Espagnat [18], we will call the former an "improper mixture", and the latter a "proper mixture". This distinction is not without some controversy, but will be found convenient for discussing different models addressing the measurement problem. In particular, decoherence in standard quantum mechanics by itself would imply that all mixtures are "improper". Whereas this can effectively account for non-selective measurements, it encounters some difficulty in accounting for individual selective measurements, which are conditioned on a read-out: namely, why all diagonal elements of the reduced density matrix except one vanish in a given single measurement episode with a known outcome? In a given measurement whose outcome has been read-out and is known (which is what measurement is all about), the measured system $S$ is formally represented by a pure state. Since the reduced density operator for an entangled system is necessarily mixed, a pure state cannot be entangled with any other system, known or unknown. This line of reasoning suggests that a procedure like Eq. (3), although able to account for non-selective measurements, is unable to account for selective measurements. A more detailed discussion of the problem is considered by Adler [16].

One other possible solution to this situation is to invoke the relative-state [21] or many-worlds [22] or similar interpretation of quantum measurement. The relative state interpretation [21] of quantum measurement rejects the projection postulate, instead postulating that each entangled $S M+$ observer correlated branch in Eq. (1) is realized in a different version of reality. Applying it to the decohered system, one argues that a given state $|i\rangle\left|\xi_{i}\right\rangle\left|e_{i}\right\rangle$ of $S M Q$ is pure relative to the observer in a given version of the universe, but mixed across the different versions 
in the multiverse. Thus the mixture $\hat{\rho}_{S M}$ resulting from decoherence is to be understood in the special sense as existing due to entanglement across the multiverse, to be conceptually contrasted from a conventional mixture of pure states existing in the given same version of the universe. The main difficulty with the Everett theory is that it is not clear how it is supposed to account for the apparent determinateness of measurement outcomes [23], while accounting for the statistical nature of measurement outcomes 24]. Attempts to reconstruct the Everett theory to address these problems have led to detailed, interesting formulations of quantum mechanics as the many-worlds [22], many-minds [25], consistent or decoherent histories [26, 27, 28], and relative-facts theories [29]. Some difficulties in their interpretation have been discussed [30]. Other novel solutions proposed include the restricted path-integral method [31] and a Grover search-based method 32.

The Bohmian model [33] offers a different but related mathematical formalism from standard quantum mechanics, based on a nonlocal deterministic picture, in which there is no discontinuity between ordinary evolution and measurement. In the collapse or dynamical program, 34, 35] and the gravitational model by Penrose [36], the projection postulate is derived as a consequence of additional physics. In the former, this is achieved via a small stochastic, nonlinear term added to the dynamical equation of the standard theory; in the latter, via an energy uncertainty due to gravitational field superpositions due to states that are spatially apart.

The present work aims to resolve the measurement problem within the decoherence scenario, by examining the information theoretic foundations of physics. The model of quantum measurement proposed here is primarily based on the premise that the content and evolution of physical phenomena are well characterized as an abstract registration and processing of quantum information, not all of which is physically accessible, on account of limitations imposed by quantum uncertainty; and that, from a certain point of view, such an approach can be as useful to describe physical phenomena as the conventional mathematical language of differential equations. We begin by calculating bounds on the quantum information processing involved in the evolution of quantum states in a Hilbert space with finite finegraining. As the next step, we study the implications of these bounds exceeding finite memory and computational resources available to physical systems, as determined by the system's energy and Hilbert space resolution. We suggest that the measurement problem finds a natural resolution as a consequence of the finiteness of quantum computational parameters characterizing physical dynamics. Thereby, the quantum to classical transition emerges as a manifestation of the underlying computational structure of physical systems.

It is of interest to note that in a number of recent works 37, 38, 39, 40, 41], bounds on the amount of information processing that can be performed by physical systems have been obtained. In particular, Lloyd [37] employs the Margolus-Levitin theorem [42] to show that the number of elementary logical operations per second ("op/s") that a physical system can perform is limited by its energy, while the amount of information that it can register bounded by its maximum entropy [43, 44]. Sivaram [39] studies the implications of model independent features of quantum gravity for everyday physics problems like field strengths, temperature, acceleration, particle energies and the performance of clock and computer performance. $\mathrm{Ng}$ [40] has argued that the product of speed and clock rate in a computer is bounded above by $t_{P}^{-2}$, where $t_{P}=\sqrt{\hbar G / c^{5}}=5.4 \times 10^{-44} \mathrm{sec}$ is the Planck time. Pati et al. 41] show that the total number of operations performed by a computer is determined by the type of interactions present in it, a fact useful in designing and building a quantum computer.

To be precise, the information dealt with in the preceding works is the accessible and useful information. Because of quantum uncertainty, this quantity is much smaller than the information needed to support or describe physical states of many-particle systems. An attempt to access this larger information in a quantum system in general 'collapses' the state, and only a small fraction, bounded by the von Neumann information [5, [6], can actually be extracted for useful information tasks.

The bounds in the numbers of op/s and bits we obtain can be interpreted in three distinct ways: (i) They give upper bounds to the amount of quantum computation that physical systems perform; (ii) they give lower bounds to the number of op/s and bits required to simulate physical systems; (iii) If one chooses to regard nature as performing computations, these numbers give the $\mathrm{op} / \mathrm{s}$ and bits in that computation. In particular, this last idea is used to motivate the 'reduction' of the state vector under measurement, and the emergence of classicality at the macroscopic level. In Sections [I and [II] we first obtain bounds on the amount of quantum information processing needed to support physical systems, and the implications of entanglement on these bounds in Section [IV] In Sections $\nabla$ and VI we present a computational model of measurement in which the observed classicality of measurement outcomes is related to parameters characterizing the information processing capacity of physical systems. This is specifically applied to projective measurements in Section VII The related issue of emergence of classicality in the macroscopic world and that of macroscopic quantum interference effects are dealt with in Section VIII]

We feel obliged to apologize in advance for the many instances of usage of computer theoretic descriptions of purely physical processes, sometimes accompanied with only qualitative justification, that the reader might encounter in this article. Yet, we believe our approach is justified, as the present model is basically about applying computer/information theoretic intuition to physical processes. Expositions of issues at a qualitative level are sometimes unavoidable, since, as we saw, some subtle issues surrounding the measurement problem hinge basically on physical 
interpretation.

\section{MEMORY ALLOCATION DEPENDS ON HILBERT SPACE RESOLUTION}

The amount of classical information required to specify even the simplest quantum system, a qubit, is obviously infinite. More generally, we can say that the amount of information (in bits) required to prepare or specify a quantum state vector- the preparation information or state information- depends on the chosen fine-graining of the Hilbert space $\mathcal{H}$ in which the quantum state vector resides. The chosen precision at which we want to resolve a system's $\mathcal{H}$ is quantified by $\mu$, the number of bits per probability amplitude needed to represent a state vector. Of these, $\mu / 2$ bits specify the real part, and the remaining $\mu / 2$ bits the imaginary part.

In the geometric formulation of the Hilbert space [46, 47], the minimum seperation between two microstates (i.e, state vectors) is given by $\phi=2^{-\mu / 2}$, where $\phi$ is the smallest resolvable Hilbert space angle, a measure of distance given by the Fubini-Study metric, a Riemannian metric defined on projective Hilbert space [48]. Eg., for a qubit specified at $\mu=2$ bytes precision, state vector resolution is a "sphere" of size $2^{-\mu} \pi \simeq 4.8 \times 10^{-5}$ radians. For a uniform ensemble of all resolvable state vectors $|j\rangle$, each with probability $p_{j}=2^{-\mu}$, on average and $D \equiv \operatorname{dim} \mathcal{H}$, the quantity:

$$
H(\tilde{p}) \equiv-\sum_{j=1}^{D-1} p_{j} \log p_{j}=(D-1) \mu \text { bits }
$$

is the state information required to construct an arbitrary $|j\rangle$. In Eq. (4), $\log ()$ refers to base-2 logarithm. In general, $H(\tilde{p}) \geq S(\rho)$, the von Neumann entropy [5, 6], because $S(\rho)$ measures entropy with basis states as the statistical alternatives, whereas $H(\tilde{p})$ measures entropy with all resolvable state vectors as the alternatives. Therefore, $H(\tilde{p})=S(\rho)$ when the ensemble consists only of orthogonal states.

For an isolated system of $D$ (finite) dimensions, the number of bits sufficient to specify the state fully at $\mu$-bit precision is given by Eq. (4), in view of the normalization condition. However, for our purpose, it is preferable to write down $\mathcal{M}$, the memory required to register the state information at any time, as

$$
\mathcal{M}=D \mu \text { bits. }
$$

This turns out to be the more efficient specification taking into consideration the time evolution of a quantum state, discussed in the next two Sections. The additional computational complexity for evaluating an amplitude via the normalization condition at each step goes as $\sim O\left(D^{n}\right) \mu$, which increases exponentially for a system of $n$ objects, whereas unitary evolution of the amplitude requires only $\sim O(\mu)$ steps. Thus the exponential increase in computational complexity that would result with the saving in memory outweighs the linear increase in memory usage. The basis in which the $D$ amplitudes are taken to be specified is some fixed, arbitrary reference basis. Later we will find that because of the finiteness of $\mu$ and decoherence, a preferred basis can emerge at the macroscopic level.

Although we have derived $\mathcal{M}$ as a Shannon information for microstates in Hilbert space, in fact it is better viewed as absolute information in the sense of Kolmogorov-Chaitin complexity [4]. Based on a modern notion of randomness dealing with the quantity of incompressible information in individual objects, their algorithmic information is a form of absolute information contained in them. It is a measure of pointwise randomness rather than average randomness produced by a source, which is the primary concern of Shannon information and its quantum generalization, von Neumann information. Thus, algorithmic information characterizes a system in a way impossible to classical probability theory (a branch of measure theory satisfying the Kolmogorov axioms). It employs the notion of the length of the shortest effective description of an individual object, which is its Kolmogorov-Chaitin complexity, to quantify the randomness of individual objects in an objective and absolute manner. We characterize $\mathcal{M}$ as the algorithmic information of the state vector. It is absolute because it depends only on $D$ and $\mu$, and not on the particular state or mixedness of the state. Thus, the pure states $|0\rangle, \cos \theta|0\rangle+\sin \theta|1\rangle$ and the maximally mixed state $(1 / 2)(|0\rangle\langle 0|+| 1\rangle\langle 1|)$ all require the same $\mathcal{M}$ : namely, $2 \mu$. This basically reflects the idea that from the viewpoint of nature, viewed as the underlying information processor, all states are pure. It is characteristic of quantum mechanics that the maximum information accessible is far smaller than $\mathcal{M}$, restricted in fact by the Holevo bound [5, 6].

For 'high fidelity' evolution, the precision parameter $\mu$ should be sufficiently large in relation to the dimension of $\mathcal{H}$. In particular, consider a uniform superposition in some basis. Each eigenstate has a probability $D^{-1}$ of being found. Each amplitude, and hence probability, is specified with accuracy $\mu / 2$. According to the computational completeness principle, the fine-graining should be large enough to specify a uniform superposition, i.e., $D^{-1}>2^{-\mu / 2}$, or $2 \log D<\mu$. In practice, for reasonably accurate state representation, we require $\mu \gg 2 \log D$, but we will use the above weaker condition as a thumb rule. 
We define total state information of an elementary particle as the algorithmic information required to specify the complex wavefunction $\psi(x, t, \Gamma)$ of a physical system over a lattice of Planck-sized cells filling all configuration space. Here $\Gamma$ represents the internal degrees of freedom. Note that the choice of position basis is quite arbitrary, and one could as well evaluate $\mathcal{M}$ using the momentum basis wavefunction $\psi(p, t, \Gamma)$. Let us consider the state information for a free electron, treated as an elementary spinor particle. In position basis, the spatial part of its state is written: $|\psi\rangle=\sum_{x=1}^{\mathcal{N}} f_{x}|x\rangle$ with $\sum_{x=1}^{\mathcal{N}}\left|f_{x}\right|^{2}=1$, where $|x\rangle$ is the position eigenstate for a particle being localized at Planck cell $x$ in the universe, $\mathcal{N}$ is the number of $l_{P}^{3}$ sized cells given by $(4 \pi / 3)\left(c t_{U} / l_{P}\right)^{3} \approx 2.8 \times 10^{181} \mu$, where $t_{U}=10^{17}$ $\mathrm{s}$ is the lifetime of the universe. Taking into consideration the spin part, we have $D=2 \mathcal{N} \approx 10^{182}$. Therefore $\mathcal{M}=D \mu=10^{182} \mu \approx 2^{600} \mu$ bits. Therefore, according to the computational completeness principle, we must have $\mu>600$ bits. It is interesting that this criterion requires us to relate a cosmological number with the property of an electron's state representation, somewhat reminiscent of Dirac's large number hypothesis [38].

Though $\mathcal{M}$ is a measure of the complexity of a system, it is different from the number of states $\exp \left(S / k_{B}\right)$, estimated from thermodynamic entropy $S$ or density of states estimated quantum mechanically. For $\mathcal{M}$ not only quantifies the number of states available to a system, but also takes into the consideration the spatial information needed to specify those states. Thus, as a rule $\mathcal{M} \geq \exp \left(S / k_{B}\right)$. In view of this, one might ask whether a measure of complexity based on a suitable conventional definition of entropy can be considered as a compressed, and hence more economic, form of the $\mathcal{M}$. The answer is two-fold: in most cases where density of states is evaluated, we often assume simplifying symmetries and time-independence. A given state (eg., the $S^{1}$ orbital in the $\mathrm{H}$ atom) can be deformed in a large number of ways as the atom interacts with its environment, none of which can be accounted for in a counting system that numbers only states. Moreover, from a computer theoretic viewpoint, which is relevant to the present model, to compute the dynamics at each time slice from such compressed information requires that the information be uncompressed at each time slice to compute the further dynamics. As explained earlier, this invariably implies an exponential increase in computational complexity. For these reasons, state information as defined above, is suitable for our purpose.

The state information may be contrasted from the amount of information that a physical system (a) can in principle register, and (b) the quantity of information that it is possible in practice to store and retrieve from a physical system, taking into consideration noise temperature, cost of transporting a bit, etc. [37, 43, 49]. As computed earlier, such a particle requires $\mathcal{M} \geq 2 \mathcal{N} \mu=2^{600} \mu$ bits of state information. As to (a), it is the von Neumann entropy bounded above by $\log D$, where $D$ is the dimension of the relevant Hilbert space. A free electron can in principle encode for $\log (2 \mathcal{N}) \approx 600$ bits. As to (b), it is $I=S / k_{B} \ln (2)=3 / 2 \ln (2)$ bits, where $S=k_{B} T d / 2$ is entropy, and $k_{B}=1.38 \times 10^{-23} \mathrm{~J} / \mathrm{K}, T, d$ are, respectively, Boltzmann constant, temperature of system, and degrees of freedom available to it. Eg., the maximum information registrable in a particle's kinetic energy is $3 / 2$ bits. These two informational quantities are far smaller than $\mathcal{M}$, which is not entirely accessible because of quantum uncertainty and quantum no-cloning [50].

It is instructive to compare $\mathcal{M}$ for a free elementary particle with the information registered by conventional computers. Estimating the total number of computers to be $\approx 10^{9}$, each registering $10^{12}$ bits, yields $10^{21}$ bits, which is vastly less than the $\approx 10^{182} \mu$ bits of state information required to completely specify a single free elementary particle. Indeed, this even exceeds the usable number of bits available in the entire universe, which is about $10^{120}$ bits, including the gravitational degrees of freedom [38].

\section{COMPUTATIONAL SPEED DEPENDS ON ENERGY AND RESOLUTION}

The maximum number of operations that can be performed by a physical system is proportional to its energy 37, 42]. This result follows essentially from the observation that the speed with which a quantum system evolves is determined by its Hamiltonian, and hence its average energy. As an example, consider the evolution of a qubit with logical states $|0\rangle$ and $|1\rangle$ on which we perform the NOT operation. To flip the qubit one can apply a potential $\hat{H}_{0}=E_{0}\left|E_{0}\right\rangle\left\langle E_{0}\left|+E_{1}\right| E_{1}\right\rangle\left\langle E_{1}\right|$ with energy eigenstates $\left|E_{0}\right\rangle=(1 / \sqrt{2})(|0\rangle+|1\rangle)$ and $\left|E_{1}\right\rangle=(1 / \sqrt{2})(|0\rangle-|1\rangle)$. Because $|0\rangle=(1 / \sqrt{2})\left(\left|E_{0}\right\rangle+\left|E_{1}\right\rangle\right)$ and $|1\rangle=(1 / \sqrt{2})\left(\left|E_{0}\right\rangle-\left|E_{1}\right\rangle\right)$, each logical state has an energy spread $\Delta E=\left(E_{1}-E_{0}\right) / 2$. Under application of the potential, the system prepared in state $|0\rangle$ for a small time $t$ becomes:

$$
|\Psi(t)\rangle=\frac{1}{\sqrt{2}}\left(\left|E_{0}\right\rangle+e^{i 2 \Delta E t / \hbar}\left|E_{1}\right\rangle\right) .
$$

It follows from Eq. (6) that the time taken to flip the qubit to $|1\rangle$ is given by $\pi \hbar / 2 \Delta E$. Similarly, it is easy to verify that after time $\pi \hbar / 2 \Delta E$, the qubit evolves so that $|1\rangle \longrightarrow|0\rangle$. Now the states $|0\rangle$ and $|1\rangle$ are mutually orthogonal. The average energy $E$ for both states $\left\langle 0\left|\hat{H}_{0}\right| 0\right\rangle=\left\langle 1\left|\hat{H}_{0}\right| 1\right\rangle=\left(E_{0}+E_{1}\right) / 2=E_{0}+\Delta E$. Thus, both the average energy and spread in energy limit the computational speed of a physical computer. By embedding a Toffoli or ControlledControlled-NOT gate in a quantum context, one can similar show that the AND and FANOUT gates, and thus a 
universal set of gates for classical computation, can be performed at a rate $f=2 E / \pi \hbar$. Therefore, the rate at which a quantum computer processes classical information is $f=2 E / \pi \hbar$, where we have set $E_{0}=0$, so that $\Delta E=E$ [42]. Here, by 'classical' we mean information recorded in orthogonal states. In principle, such information can be encoded, processed and read-out with complete certainty.

The evolution of state information is clearly much faster. Consider a $D$-dimensional system, driven by the Hamiltonian $\sum_{i=1}^{D} E_{i}\left|E_{i}\right\rangle\left\langle E_{i}\right|$. Along any given 'direction' in Hilbert space, the minimum distance is $\phi=2^{-\mu / 2}$. The minimum time to traverse from one microstate to the neighboring is $2^{-\mu / 2} \hbar / E_{i}$. Thus, the $i$ th amplitude evolves at the speed $f(i)=2^{\mu / 2} E_{i} / \hbar$. Therefore, the speed in op/s at which amplitudes are in effect computed as a physical system evolves in real time is:

$$
f_{q}=\frac{2^{\mu / 2} D E}{\hbar} \mathrm{op} / \mathrm{s}
$$

where average energy $E=\sum_{i} E_{i} / D$. We can view $f_{q}$ as the bit rate at which $D$ amplitudes of a system with energy $E$ are being evolved at $\mu$-bit precision. For a two-state system $f_{q}=2^{\mu / 2} \pi f$. The implicit quantum information processing proceeds exponentially faster in $\mu$ than usable classical information processing.

Applying this result to an electron with energy $E=m c^{2} \approx 8.2 \times 10^{-14} J$ shows that an electron can in principle process classical information at the rate $f=8.6 \times 10^{20}$. If the electron is treated as a qubit, with two dimensions, and amplitudes are specified at double precision, i.e., $\mu=64$ bits, then the speed of computation implied by an isolated electron's evolution is $f_{q}=2^{32} f=3 \times 10^{30}$ elementary logical operations per second. More realistically, if the electron is treated as an elementary spinor particle, the speed of computation is $f_{q}=10^{\mu / 2} 10^{182}\left(8.2 \times 10^{-14}\right) /\left(6.1 \times 10^{-34}\right) \approx$ $10^{202} 2^{\mu / 2} \mathrm{op} / \mathrm{s}$. Assuming that amplitudes are specified with the minimal 600 bit-precision, $f_{q}=10^{202} 2^{300} \approx 10^{292}$ $\mathrm{op} / \mathrm{s}$.

Interestingly, the computational speed of all conventional computers combined is far lesser than the number of logical operations performed in the quantum evolution of an single electron. With about $\approx 10^{9}$ computers operating at a clock rate of $\approx 10^{9} \mathrm{~Hz}$ performing $\approx 10^{5}$ elementary logical operations per clock cycle, all the human-made computers in the world are operating at no more than $10^{23} \mathrm{op} / \mathrm{s}$. This is only 3 orders larger than the rate at which classical logical operations are preformed by a single electron, but about 270 orders smaller than processing rate corresponding to the evolution of an electron at the minimal, 600-bit resolution of $\mathcal{H}$. In this and the previous Section, we saw that energy and Hilbert space resolution limit the computational speed and memory support required as a quantum system evolves. In the following Section, we will find that these needs are exponentially augmented in the presence of entanglement.

\section{ENTANGLEMENT AUGMENTS SPEED AND MEMORY REQUIREMENT}

In practice, few physical things in the universe are truely isolated. Systems are constantly interacting and, as a result becoming entangled. Consider the interaction of $n D$-dimensional objects, each characterized by Hilbert space $\mathcal{H}_{D}$. When isolated, each has a memory requirement $\mathcal{M}=D \mu$ bits. If they do not interact, and thus remain separable, their total memory requirement is simply $n D \mu$ bits. But if they do, they will in general become entangled, and the combined system is characterized by the $D^{n}$-dimensional Hilbert space $\mathcal{H}_{D}^{\otimes n}$, so that the general state is now described by the exponentially larger $D^{n}$ amplitudes. This is the familiar consequence of the tensor product character of Hilbert spaces [5, 6], and reflects the fact that multiparticle systems, having more permutations of configuration, are exponentially more complex than isolated systems. The memory requirement for the system, composed of elementary objects, is given by

$$
\mathcal{M}=\mu D^{n} \equiv \mu \mathcal{D} \text { bits. }
$$

If the dimensions of the interacting elementay objects is $D_{1}, D_{2}, \cdots$, then $\mathcal{D}=\Pi_{i} D_{i}$, and $\mathcal{M}=\mathcal{D} \mu$. On the other hand, if the objects are non-interacting, and thus remain separable, then $\mathcal{M}=\left(\sum_{i} D_{i}\right) \mu$.

One might very loosely regard $\mathcal{M}$ as a measure of entanglement in the system in the limited sense that given a $n$-partite state, it is larger for an entangled state than for a separable state. However, it is an absolute quantity, depending only on $n, D$ and $\mu$, and not on the particular state or mixedness of the state. Entangled states with different conventional quantifications of entanglement can have same the $\mathcal{M}$, whilst states quantified with the same entanglement conventionally can have different $\mathcal{M}$. For example, the states $(1 / \sqrt{2})(|00\rangle+|11\rangle)$ and $\cos \theta_{1} \cos \theta_{2}|00\rangle+$ $\cos \theta_{1} \sin \theta_{2}|01\rangle+\sin \theta_{1} \cos \theta_{3}|10\rangle+\sin \theta_{1} \sin \theta_{3}|11\rangle\left(\theta_{1}=\pi / 3, \theta_{2}=-\theta_{3}=\pi / 4\right)$ are quantified to have two different amounts of (entropy of) entanglement [3] ( 1 bit and 0.81 bits, respectively), but their state information is stored using the same amount of memory: $\mathcal{M}=4 \mu$ bits. This reflects the fact that when $n$ objects are entangled, $\Pi_{i} D_{i}$ amplitudes 
are required to fully specify the state, irrespective of their particular values: whether some of them have null value or such as to make the state maximally entangled. Being absolute, $\mathcal{M}$ depends on the actual ensemble of state vectors, and not only the density operator. By definition, a uniform mixture of 24 -state systems in the separable states $\mathcal{E}_{1} \equiv\{|00\rangle,|11\rangle,|22\rangle,|33\rangle\}$ has the same amount of entanglement as a uniform mixture of entangled states: $\mathcal{E}_{2} \equiv\{(1 / \sqrt{2})(|00\rangle+|11\rangle),(1 / \sqrt{2})(|00\rangle-|11\rangle),(1 / \sqrt{2})(|22\rangle+|33\rangle),(1 / \sqrt{2})(|22\rangle-|33\rangle)\}$, namely zero. In fact, the two mixtures are indistinguishable, having the same density matrix. Yet, we have $\mathcal{M}\left(\mathcal{E}_{1}\right)=2 \times 4 \mu=8 \mu$ bits and $\mathcal{M}\left(\mathcal{E}_{2}\right)=4^{2} \mu=16 \mu$ bits. This reflects the idea that to nature, viewed as the underlying information processor, all states are pure, and the entanglement depends on the individual states in an ensemble and not on the density matrix.

The information processing resource Eq. (8) presupposes is very large. Consider a "laptop" of mass $m=1 \mathrm{~kg}$ and registering information in the spin of the particles composing it. The total number of baryons, and therefore the total number $n$ of bits registrable therein, is about $n \equiv 1 / m_{p} \approx 6 . \times 10^{26}$ bits. The memory $\mathcal{M}$ that can support the evolution of this many qubits is the superastronomical number $2^{6 . \times 10^{26}} \mu \approx 10^{1.81 \times 10^{26}} \mu$ bits, where $\mu>2$, according to the computational completeness principle. (It is worth noting that the number is $10^{\left(1.81 \times 10^{26}\right)}$, and not the much smaller $\left(10^{1.81 \times 10}\right)^{26} \approx 10^{260}$; i.e., $\log _{10}(\mathcal{M}) \sim O\left(10^{26}\right)$ ). Not surprisingly, conventional memory in the entire universe, let alone the part available to conventional computers, will hardly suffice to support the information processing overhead corresponding to the evolution of such a quantum system. Suppose Moore's law has been taken to its possible extreme, whereby all particles in the universe register a bit each. Estimating the number of baryons in the universe at $\beta=10^{76}$ (cf. below), we find using Eq. (8) that the most number of qubits $(D=2)$ whose state information can be registered is $n=76 \cdot \log _{2}(10)-\log _{2}(64) \approx 246$ qubits. Thus, the entire accessible world is not enough to track the state of even 300 qubits at just double precision. In fact, the Bekenstein bound [4], together with the holographic principle [51], implies that the maximum information that can be registered in a system is $A / l_{P}^{2}$, where $A$ is the system's area. Applying this to the universe as a whole implies that the maximum number of (classical) bits that could be registered by the universe using matter, energy and gravity is $c^{2} t_{U}^{2} / l_{P}^{2} \approx 10^{120}$ bits [38]. Solving for $n$ in Eq. (8) with $\mathcal{M}=10^{120}$ bits and $\mu=64$ bits yields 398.6 qubits. In other words, the maximum possible information registrable in the universe is insufficient to record the state information for more than 400 qubits.

Coming to the continuous case, let us consider $n$ 2-state particles in a box of volume $V$. The memory support required for a single particle is $\mathcal{M}=2(4 \pi / 3)\left(c t_{U} / l_{P}\right)^{3} \mu \approx 10^{182} \mu$ bits, where the wavefunction has null value for regions outside the box. The memory support for $n$ particles is, as argued above,

$$
\mathcal{M}=\left[2(4 \pi / 3)\left(c t_{U} / l_{P}\right)^{3}\right]^{n} \mu \approx 10^{182 n} \mu \text { bits. }
$$

It would appear that Eq. (9) in fact applies to distinguishable particles, with that for indistinguishable particles given by $\mathcal{M}=\left\{2(4 \pi / 3)\left(c^{3} t_{U}^{3} / n V_{P}\right)\right\}^{n}$, since each particle has effectively $(4 \pi / 3) c^{3} t_{U}^{3} / n$ volume to be in. However, because $\mathcal{M}$ is an absolute quantity, it keeps track of individual particles, whether or not they are distinguishable quantum mechanically. Thus, the former expression is appropriate. Let us consider the $1 \mathrm{~kg}$ laptop. If made up of $\mathrm{H}$ atoms, it is constituted of $n=1 / m_{p} \approx 6 . \times 10^{26}$ baryons, or about $4 n$ quarks and electrons, assumed to be elementary paticles. Thus $\mathcal{D} \approx\left(10^{182}\right)^{4 n}=10^{1.82 \times 10^{29}}$, so that $\mathcal{M} \approx 10^{1.82 \times 10^{29}} \mu$ bits.

It is instructive to contrast this with maximum information $\mathcal{M}$ that would be needed, for an 'ultimate laptop' weighing $1 \mathrm{~kg}$ and occupying 1 liter volume. This corresponds to the maximized entropy of the laptop obtained by converting all its matter into radiation [37]. The conventional equation for blackbody radiation can then be used to estimate the temperature $T$ that would be obtained if that matter were converted to radiation at temperature $T: \rho c^{2}=\left(\pi^{2} / 15 \hbar^{3} c^{3}\right)\left(k_{B} T\right)^{4}$. Solving for the temperature gives $k_{B} T=\left(15 \hbar^{3} c^{5} \rho / \pi^{2}\right)^{1 / 4}$. The number of photons is estimated as $2 \rho c^{2} / 3 k_{B} T=(2 / 3 \ln (2))\left(\pi^{2} V / 15 \hbar^{3} c^{3}\right)^{1 / 4} E^{3 / 4}$, which, for the ultimate laptop yields the value of $n=1.6 \times 10^{31}$ particles [37]. We then have $\mathcal{D}=\left(10^{182}\right)^{n} \approx 10^{4.4 \times 10^{33}}$, so that $\mathcal{M}=10^{4.4 \times 10^{33}} \mu$ bits of memory correspond to the quantum state of the ultimate laptop.

Let us consider the action of the Hamiltonian $\hat{H}=\sum_{j=1}^{n} \hbar \omega_{j} \hat{\sigma}_{x}^{\otimes j}$ on a system of $n$ qubits initially prepared in the state $|0\rangle^{\otimes n}$, i.e., each qubit in an eigenstate of the Pauli operator $\hat{\sigma}_{z}$. Note that, being separable, it requires in general just $2 n \mu$ bits to be specified. But, after time $t$ they will have evolved, by the action of $e^{-i \hat{H} t}$, to the entangled state:

$$
|\Psi(t)\rangle=\frac{1}{2^{n / 2}} \sum_{k=0}^{2^{n}-1} \sum_{j=1}^{n} e^{-i \omega_{j}\left(1-2 \pi_{k}(j)\right) t}|k\rangle,
$$

where $\pi_{k}(j)$ is the parity of the first $j$ digits in the binary representation of string $k$. Thus, we now need $2^{n}$ (though not all independent) amplitudes to describe the entangled state. It is not difficult to see that, for sufficiently intricate interactions, all $2^{n}$ amplitudes will be independent. More importantly, as noted earlier, the fact of entanglement between the $n$ objects, by itself, irrespective of the magnitude of entanglement, is the relevant quantity to quantify $\mathcal{M}$, which in this case is $2^{n} \mu$ bits. 
Now suppose that at $t=t_{0}$, the interaction Hamiltonian is switched off, and the system $\left|\Psi\left(t_{0}\right)\right\rangle$ is subjected to (free) evolution with the Hamiltonian $\hat{H}_{1}=\sum_{j=1}^{n} \hat{H}_{0}^{(j)}$. Applying the action $e^{-i \hat{H}_{1} t}$ for time $\Delta t$, we find that the state $\left|\Psi\left(t_{0}\right)\right\rangle$ evolves to:

$$
\left|\Psi\left(t_{0}+\Delta t\right)\right\rangle=\sum_{k=0}^{2^{n}-1} e^{-i\left(\Omega(k) \Delta t+p(k) t_{0}\right)}|k\rangle,
$$

where $\Omega(k)=n_{k}(0) E_{0}+n_{k}(1) E_{1}$, where $n_{k}(i)$ is the number of $i$ 's in string $k$, and from Eq. (10), we have $p(k)=$ $\sum_{j=1}^{n} e^{-i \omega_{j}\left(1-2 \pi_{k}(j)\right) t_{0}}$. The average energy of the state is $\left\langle\Psi\left(t_{0}+\Delta t\right)\left|H_{1}\right| \Psi\left(t_{0}+\Delta t\right)\right\rangle=n\left(E_{1}+E_{2}\right) / 2=n E$. Each amplitude is evolving with average $\bar{\Omega}=n E$. This shows that, for the generic entangled state, the speed of evolution of the whole system is governed by the average energy of the whole system. Therefore, the computational speed $f_{q n}$ at which a system of $n D$-dimensional objects of average energy $E$ is got by inserting the appropriate values in Eq. (7):

$$
f_{q n}=\frac{2^{\mu / 2} D^{n} n E}{\hbar} \equiv \frac{2^{\mu / 2} \mathcal{D E}}{\hbar} \mathrm{op} / \mathrm{s}
$$

where $\mathcal{E}=n E$ is the average energy of the whole system. We can view $f_{q n}$ as the bit rate at which $D^{n}$ amplitudes of a system with energy $n E$ are being evolved at $\mu$-bit precision. The clock rate $\omega$ for a system is defined as the computational speed per bit, given by $\omega=f_{q n} / \mathcal{M}=2^{\mu / 2} n E / \mu \hbar$.

The computational speed required to sustain the evolution of the $1 \mathrm{~kg}$ laptop of 1 liter is, according to Eq. (12), $f_{q n}=\left(2^{\mu / 2} m c^{2} / \hbar\right) \cdot 10^{1.82 \times 10^{29}} \approx 2^{\mu / 2} 10^{50+1.82 \times 10^{29}} \mathrm{op} / \mathrm{s}$. Now, it can be shown that for a matter-dominated universe at its critical density, the total number of logical operations that can have been performed within the particle horizon at time $t_{U}$ is $\left(t_{U} / t_{P}\right)^{3} \approx 10^{120} \mathrm{op} / \mathrm{s}[38]$, where $t_{P} \equiv \sqrt{\hbar G / c^{5}}=5.391 \times 10^{-44}$ secs. Thus, we find that the entirety of in-principle accessible operations performed in the universe is insufficient to support even a second of evolution of the laptop. Put another way, if all the accessible computation performed in the universe were made available, it would have sufficed to support the evolution the laptop for no more than $\approx 10^{120} /\left(2^{\mu / 2} 10^{50+1.82 \times 10^{29}}\right) \approx 2^{-(\mu / 2)} \times 10^{70-1.82 \times 10^{28}}$ sec, which is much smaller than even $t_{P}$.

We can similarly compute the bounds for the universe as a whole: all matter and radiation in the universe was fashioned from its primordial content, coming from Milky Way matter, which ultimately originated in the Big Bang over $10^{17}$ sec ago. Hence, the worldlines of all objects in the universe were enmeshed in that distant past, and in principle, the entire universe is a closed, entangled system. If we subscribe to the standard view that evolution is purely unitary, then, inspite of the apparent classical behavior of the visible world, there is a minute amplitude entangling all nearby objects to matter in the distant stars and beyond. As before, suppose all matter is converted into radiation. The resulting number $n$ of photons is about $2 \rho c^{2} / 3 k_{B} T=(2 / 3 \ln (2))\left(\pi^{2} V / 15 \hbar^{3} c^{3}\right)^{1 / 4} E^{3 / 4} \approx 10^{90}$, where $\rho=10^{-27}$ $\mathrm{kg} / \mathrm{m}^{3}$ corresponding to one proton $/ \mathrm{m}^{3}$. We then have $\mathcal{D} \approx\left(10^{182}\right)^{n} \approx 10^{1.82 \times 10^{92}}$, so that $\mathcal{M}=10^{1.82 \times 10^{92}} \mu$ bits of memory are required to record the state of the entire universe. The evolution of the universe is supported by a computational speed $f_{q n}=2^{\mu / 2} \cdot 10^{1.82 \times 10^{92}} E / \pi \hbar=2^{\mu / 2} \times 10^{100+1.82 \times 10^{92}}$ op/s according to Eq. (77).

\section{WHAT IS NATURE COMPUTING?}

The above sections calculated how many elementary operations and how much memory are equivalent to the state and evolution of some simple physical systems. As noted earlier, there are three distinct interpretations of the numbers calculated. The first interpretation simply states that the number of op/s and number of bits given here are upper bounds to the amount of quantum computation that are performed during the course of evolution of a physical system. This interpretation should be uncontroversial: a particle that is passed through a double-slit, for example, can be regarded as a quantum computer that computes the Fourier transform of the slit configuration. Since the effect arises because of the interference of amplitudes, this clearly establishes that, even if the path taken by a quantum object is not an element of physical reality in the Einstein-Podolsky-Rosen sense [52], computations are performed during the evolution of the amplitudes to effect the Fourier transformation. A more complicated example, involving entanglement, as against single particle superposition, is the Shor algorithm, involving the computation of the periodicities of certain modular functions [53]. Here again we are reminded in a dramatic way that computations are performed in all $D^{n}$ dimensions, even though in a given run, we can access only a much smaller quantity.

The second interpretation of $\mathcal{M}$ and $f_{q n}$ notes that the numbers calculated give a lower bound on the number of bits needed and op/s that must be performed by a quantum computer that performs a direct simulation of a physical system at some fixed resolution of $\mathcal{H}$. This interpretation should also be uncontroversial: it is well recognized that 
quantum computers are better than classical computers in certain tasks, specifically simulating quantum systems, because the former can compute with massive parallelism determined by the dimension and entanglement of the system [54]. The true of quantification of $\mathcal{M}$ and $f_{q n}$ will depend of what the actual degrees of freedom in nature are, which should determine the ultimate model of Theory of Everything (TOE) we use, whether it is of elementary strings [55], membranes or loops in quantum foam [56].

The third interpretation- that the numbers of bits and op/s calculated here represent the actual memory capacity and elementary operations performed by nature- is more controversial. It is of relevance to novel, computational models of the universe such as presented in Refs. [57, 58, 59]. If this interpretation is meaningful, then an elementary particle is best viewed simply as an information theoretic entity, in particular, a data structure - contingent regions in space where memory about a single object is concentrated. We view state information as being encoded simply in terms of specifying the wavefunction at each point in configuration space, since this is sufficient to reconstruct the entire state of the particle. The particle's (smeared) position in configuration space may be regarded as the memory address for accessing the information content of the particle. Interactions emerge in this picture as gates for accessing and/or modifying this information content. Interestingly, this picture provides a converse scenario to the fact that virtually all physical interactions can operate as logic gates [37.

That nature registers an amount of information equal to the logarithm of the number of all its possible microstates in Hilbert space seems reasonable, considering that any of them is a possible outcome of a suitably chosen observable. But whether or not it makes sense to identify the local evolution of information-carrying degrees of freedom with elementary quantum logical operations by nature is a question which the remaining part of this article is dedicated to. Indeed, we can first ask the question: What sort of evidence would be needed to demonstrate an essential computational character of nature? How does one distinguish it from other effective models of quantum mechanics? We need to clarify what a suitable answer to these questions would be. It seems that, to be free from interpretational and philosophical ambiguity, it must be able to reasonably demonstrate that certain physical phenomena are better described computationally than dynamically, eg., the appearance of certain discontinuities could be attributed to finite information processing resources supporting the evolution of physical systems. In the next and following Sections, we argue that quantum measurement provides a possible window onto such a demonstrable essential computational apparatus underlying physical reality. In particular, as explained below, the classicality of measurement outcomes and of macroscopic phenomena are manifestations of information overflow in this underlying apparatus. We believe that the computational model of quantum measurement presented here, in response to the above questions, can perhaps induce further insight into the relationship between physics and computation.

In such an interpretation: what is nature computing? If one chooses to regard nature as performing a computation, most of the elementary operations in that computation consist of position amplitudes of various particles constantly evolving, as quarks, electrons and photons move from place to place and interact with each other according to the basic laws of physics. In other words, to the extent nature is performing a computation, it is "computing" its own dynamical evolution [60]. Only a extremely small fraction of the universe is classically accessible, and an even smaller fraction being performed on conventional digital computers.

\section{INFORMATION TRANSITIONS IN LARGE PHYSICAL SYSTEMS}

To calculate the number of bits necessary and op/s that are performed during a measurement requires a model of measurement. In the initial, pre-measurement step, the measured system $S$ and the measuring apparatus $M$ interact via Hamiltonian $\hat{H}$ and become entangled, as noted in Eq. (11). For macroscopic systems, this implies large magnitudes of computational resources to support the required $\mathcal{M}$ and $f_{q n}$. In view of this, it is not unreasonable in a computational model of physical evolution to suppose that there are finite parameters and procedures to ensure efficient and error-free information processing. The question is whether/how such computational parameters and procedures will be reflected in the overlying dynamics. As seen earlier, over $10^{1.82+10^{92}} \mu$ bits are required to support the quantum evolution of the universe as an entangled whole. In an actual program that stores and accesses so many bits, computational support of the same order will be needed in the abstract operating system, introducing more complexity. For example, further working cost is incurred in creating similar-sized arrays of pointer variables in order to accurately match the exponentially increasing number of amplitudes to the corresponding multiparty eigenstates. This demands accurate tracking and error correction. Note that even if nature's information processing is noise-free in the classical sense (where noise can be introduced from the external environment), it would seem that models of physical reality built on intrinsic randomness, eg., Refs. 57, 58], are expected to require error correction in order to counter errors arising purely from random fluctuations, which can introduce Gaussian noise. Such intrinsic quantum error correction will ensure the observed regularity of the laws of physics in nature (in terms of the constancy of the fundamental constants and laws).

In the following two subsections, we examine two, independent possible scenarios for the emergence of classicality 
at a macroscropic level as a consequence of limitations imposed by the finiteness of the fine-graining of Hilbert space, which turns out to be equivalent to an effective finite size of the memory and information processing capacity of the 'operating system' underlying physical systems. The detailed implications of these scenarios for the dynamics of macroscopic systems is dealt with in Sections VII and VIII

\section{A. Quantum chaos scenario}

From communication engineering and the study of natural genetic systems [61], we know that error correction is necessary for detection and elimination of errors during information processing. It permits the safe processing of input information, and the transmission of information across space and time. Similarly, if the Schrödinger evolution of physical systems is indeed a manifestation of underlying information processing in nature based on intrinsic randomness, nature's error correction should play a role in guaranteeing the temporal and spatial symmetries of physical laws. For example, in the process physics model [57], self-referential noise gives rise to the basic structure of space and some quantum phenomena. Statistically speaking, over $n$ noisy processes, 'errors' in nature can arise with a probability $n^{-1 / 2}$. While this will be extremely small, it can in principle give rise to rare unexpected deviations from the physical laws. It is very highly unlikely that such errors in the enforcement of physical laws won't be smudged out; and, even if detected, they will be counted as a statistical quirk. Yet, in principle, the role for some sort of error correction can never be ruled out. In particular, it will be necessary to thwart the onset of quantum chaos, induced by such imperfections, even when operating in a closed system,

Now, this may seem quite unexpected considering that quantum mechanics is usually known to suppress classical chaos. Yet, based on the studies of the simulation of a generic model of a quantum computer, with qubits interacting with coupling strength $J$, Georgeot and Shepelyansky [62] find that for coupling strength $J>J_{c}$, quantum chaos emerges, induced by imperfections and residual inter-qubit coupling. It leads to ergodicity of the quantum computer eigenstates. Here the critical coupling strength $J_{c}$ is given by $J_{c} \sim \Delta_{0} / n, \Delta_{0}$ the average energy distance between the two levels of a qubit, and $n$ the number of interacting qubits. Above the quantum chaos border, an initial register state $|\psi\rangle$ will spread quickly with time over an exponential number of eigenstates of the system with residual interaction, destroying the operability of gates. With the disappearance of non-interacting qubit structure, the quantum computer as a whole suffers a melt-down [62]. In the context of the quantum computer model of the universe, this would mean that, because of strong interactions between particles, an initial random error could be chaotically amplified until physical reality sinks into an almost chaotic haze of matter and radiation, in contrast to the structured world we see around us. Such a chaotic thermalization of the universe is not conducive to the formation of stable structures, let alone complex systems like the human beings, as we know them.

In simulations of a quantum computer, the time scale in the chaotic regime after which quantum chaos sets in is given by $\tau_{X} \sim 1 / \gamma$, where, as in many-body systems, the spreading rate $\gamma$ can be estimated as $\gamma \sim n J^{2} / \delta$ for $J_{c}<J<\delta[62]$. Setting $\delta \approx J$, we write:

$$
\tau_{\chi} \approx 1 / n J
$$

which shows that the number of interacting bodies and the strength of their interaction induce a more rapid onset of chaos. In principle, the developement of quantum chaos can be suppressed by error-correcting codes applied within this time. If the error correction code in nature has a code rate $r[63]$, then $\mathcal{D} \equiv D^{n}$ possible states are encoded into and decoded from $\mathcal{D}^{\prime} \equiv D^{n / r}$ vectors. From Eq. (13), the angular frequency at which error correction must be performed is $\omega_{\chi} \geq n J / \hbar$. The information processing rate for error correction is proportional to the number $\mathcal{D}^{\prime}$ of abstract vectors. However the memory supporting the system, and into which the corrected information is "loaded", is $\mathcal{M}=\mu \mathcal{D}$ in number. Therefore, to stave off potential chaos in physical reality, each supporting bit in the underlying processor must perform operations at rate $\omega_{\chi}\left(\mu \mathcal{D}^{\prime} / \mathcal{M}\right)=\omega_{\chi} D^{n x}$ where $x=((1 / r)-1)$ [64]. It is reasonable to expect that for error correction to be fast enough to thwart the onset of quantum chaos, the above rate must not be much larger than the clock rate $\omega$ for the system. Therefore, the condition that an object's 'processor' can correct for chaotically induced errors fast enough is, $\omega>\omega_{\chi}\left(\mu \mathcal{D}^{\prime} / \mathcal{M}\right)$, or:

$$
2^{\mu / 2} n E \geq \hbar \omega_{\chi} \mu D^{n}
$$

where we approximate $x \approx 1$. For fixed $\mu$, the left-hand side increases linearly with $n$, whilst the right-hand side increases exponentially. Clearly, for sufficiently large $n$, the required error correction exeeds the clock rate available to the physical object. Such a large object can no longer process its error correction quickly enough to stave off the onset of chaos, without experiencing a 'burn-out'. An object for which the inequality (14) is violated is said to be computationally unstable. 
Using Eq. (8), we obtain an inequality equivalent to Eq. (14):

$$
\mathcal{M}<2^{\mu / 2}\left[\frac{E}{J}\right] \equiv \mathcal{M}_{1} \text { bits }
$$

where $E / J$ is the ratio of the average energy to coupling strength for each object in the system. Effectively, $\mathcal{M}_{1}$ can be regarded as the finite upper limit to the amount of memory available to a system. A sufficiently large, complex system for which $\mathcal{M}>\mathcal{M}_{1}$ is computationally unstable because it is trying to access memory not allocated to it. The situation will lead to a situation akin to a 'segmentation fault' [65] in conventional computing, which occurs when a computer program tries to access memory locations not allocated for the program's use, eg., when one tries to access an array index which is out of the declared range.

Let us consider an elementary particle, initially in a separable state. In this case, $n=1$ and it is assumed that $\mu$ is sufficiently large to satisfy Eq. (14). The particle manifests pure quantum mechanical behavior, its unitary evolution being governed by Schrödinger equation (or an appropriate relativistic generalization thereof). As the particle interacts with its immediate surrounding, it rapidly becomes entangled with them, and $n$ increases. $\mathcal{M}$ grows exponentially according to Eq. (8). For sufficiently large $n$, inequality (14) is violated. The system's coherent unitary Schrödinger evolution can no longer be computationally supported for such a large- $n$ system. Error-correction can no longer be performed fast enough to ward off emergence of quantum chaos. The thus-far computationally stable system attains to computational instability.

The system's subsequent behavior, which is a key component of the model, is better understood computer theoretically than dynamically: the computationally unstable system experiences the quantum information equivalent of a 'program crash' [66], that instantaneously projects the system into a separable state of $n$ particles. Thereby it re-enforces inequality (14) and restores computational stability, since now $n=1$ for each of the $n$ disentangled particles. The memory limit $\mathcal{M}_{1}$ can thus be thought of as an automatic servo-mechanism to stave off the thermalization of the system through the onset of quantum chaos. The crash of the unitary phase leads to the quantum informational equivalent of a 'core dump' 67]. In our case, the core is generated as a snapshot of one of the $D^{n}$ separable states residing in the memory just before the crash, each specified with $\mathcal{M} \sim O(n \times \mu D)$ bits, corresponding to a mnemonically minimal state, i.e., a state that, being separable, requires minimum memory $\mathcal{M}$ to specify. The core describes the state of the system after the crash. Therefore, the crash represents an abrupt information transition from a computationally unstable state to a mnemonically minimal, computationally stable state. The term 'transition' denotes the fact the change is discontinuous, in contrast the smooth build-up of $\mathcal{M}$ is the preceding phase of unitary evolution. And the 'core dump' is equivalent to the projection in Eq. (3). It specifies the initial conditions for the subsequent unitary phase. Information transition causes the system to factor out momentarily from the rest of the universe. Loosely, the system is thereby said to have 'collapsed' non-unitarily into a random separable state.

As we find later, the basis set of mnemonically minimal states into which the unitary phase information-transitions, called the transition basis, is under many circumstances uniquely determined by environment-induced decoherence. Which particular state in the transition basis is chosen is determined probablistically to satisfy the Born rule. Whether the choice is truely random, or pseudo-random, being determined by nature's unknown underlying computational architecture, is beyond the scope of the present work. Non-selectively, the information transition turns the computationally unstable state into a proper mnemonically minimal mixture. Thus, information transitions serve as an engine for turning highly entangled states into proper mixtures. Upon the crash of the unitary phase of a closed system, memory is emptied (freed), being made available for the subsequent unitary phase. We will show in detail in Sections VII and VIII that the classical behavior of large systems arises from the very rapidly alternating sequence of unitary and (information) transition episodes.

Consider $n_{\max }$ as the value of $n$ that saturates inequality (14). Taking logarithm on both sides of Eq. (14), we see that, for $n_{\max }$ and $\mu$ sufficiently large and $J \approx E, n_{\max } \sim \mu$, i.e., the number of particles that can become entangled before a system information transitions, ie., its wavefunction 'collapses', is of the order of the precision with which Hilbert space is specified. Roughly speaking, this means that the number of particles that can get entangled without collapsing is approximately $\mu$. Or, the scale at which nature becomes classical reflects the fine graining of Hilbert space. Macroscopic systems, with $n \gg \mu$, will have their unitary evolution continually interrupted by information transitions, and hence behave classically. Microscopic systems, with relatively less complexity and degrees of freedom, for which $n \ll \mu$, being computationally stable, retain unitary evolution and hence behave quantum mechanically. For fixed fine-graining of Hilbert space, all systems larger than $n_{\max }$ are classical. Provided the universe is large enough, i.e., $n_{\max }<10^{120}$ (according to the estimate that includes gravitational degrees of freedom [38]), classicality at some sufficiently large scale is inevitable. 


\section{B. Quantum computational incompleteness scenario}

The main conclusions of the above Subsection follow also without our invoking quantum chaos, in the following way. In specific, suppose the state of a system is given by $|\Psi\rangle=\sum_{k=1}^{\mathcal{D}} A_{k}|k\rangle$, where $\mathcal{D}(\gg 1)$ is the system's dimension, and $\sum_{k=1}^{\mathcal{D}}\left|A_{k}\right|^{2}=1$. In a uniform superposition, $\left|A_{k}\right|^{2}=\mathcal{D}^{-1}$. According to the computational completeness principle, fine-graining parameter $\mu$ should be large enough to support a uniform superposition. The maximum precision at which $\left|A_{k}\right|$, and hence $\left|A_{k}\right|^{2}$, can be specified is $2^{-\mu / 2}$. Thus, we require $2^{-\mu / 2} \leq \mathcal{D}^{-1}$, or $\mu \geq 2 \log \mathcal{D}$. In particlar, if the system consists of $n D$-dimensional objects, then $\mathcal{D}=D^{n}$ so that

$$
\mu \geq 2 n \log D,
$$

for "high fidelity" evolution. Let $n_{\max }=\mu /(2 \log D)$ be $n$ that saturates inequality (16). For any fixed $\mu$, a sufficiently large system of entangled objects, namely one for which $n \gg n_{\max }$, will fail the computational completeness condition. In such a large system, an exponentially increasing number $\left(>\left(D^{n}-n_{\max }\right)\right)$ of small $\left(\left|A_{k}\right|^{2}<2^{-\mu / 2}\right)$ but finite amplitudes are being set to zero, because Hilbert space fine-graining is not fine enough. As a result, the system can no longer record amplitudes and track their evolution accurately enough to enact a high fidelity evolution. An object for which the inequality (16) is violated is said to be computationally unstable. This gives the second scenario for computational instability, apart from that discussed in subsection $\nabla 1 \mathrm{~A}$

Using Eq. (8), we can express condition (16) for computational completeness as a limit on memory available to a physical system:

$$
\mathcal{M} \leq \mu \times 2^{\mu / 2} \equiv \mathcal{M}_{2} \text { bits. }
$$

This may be compared with the expression for memory threshold $\mathcal{M}_{1}$, obtained in Eq. (15). Since the exponential dependence on $\mu$ will most dominate both expressions, these two expressions are not inconsistent with each other. In fact, $\mathcal{M}_{1} / \mathcal{M}_{2}=E / \mu J$. There is good reason to believe on this account that $\mathcal{M}_{1} \ll \mathcal{M}_{2}$. The former threshold, being smaller, will be reached faster, and thereby be the more decisive. However, the $\mathcal{M}_{2}$ scenario is simpler, and perhaps more plausible. For this reason, in future discussions, we will generally refer to $\mathcal{M}_{2}$ rather than $\mathcal{M}_{1}$. With this interpretation, we see that the evolution of a computationally unstable system is analogous to the execution of a conventional program with invalid pointer de-referencing, which will inevitably lead to a segmentation fault. A sufficiently large, complex system, for which $\mathcal{M}>\mathcal{M}_{2}$ is computationally unstable because it is trying to access memory not allocated to it.

Let us consider an elementary particle initially in a separable state, described as a system with $n=1$. As it interacts with neighboring particles, and become entangled, it is described as part of a system with increasing $n$. The memory requirement of this system grows exponentially, as given in Eq. (8). At the point where $n \gg n_{\max }$, or equivalently $\mathcal{M} \gg \mathcal{M}_{2}$, the system becomes computationally incomplete. Error correction is no longer possible, because the processor lacks the Hilbert space fine-graining needed to keep track of the exponentially diminishing amplitudes. The effective non-availability of memory for tracking all amplitudes, necessary for accurate enactment of the multi-particle system's evolution, leads to computational instablity.

As in the above burn-out scenario, the system's subsequent behavior, which is a key component of the model, is better understood computer theoretically than dynamically: the computationally unstable system experiences an information transition, that instantaneously projects the system into a separable state of $n$ particles. Thereby it re-enforces inequality (16) and restores computational completeness and stability, since now $n=1$ for each of the disentangled particles. The memory limit $\mathcal{M}_{2}$ can thus be thought of as an automatic servo-mechanism to safeguard computational completeness. Indeed, it may not be inappropriate to liken $\mathcal{M}_{2}$ to the critical temperature $T_{c}$ in phase transitioning systems. The crash of the unitary phase leads to the quantum informational equivalent of a core dump. The core is generated as a snapshot of one of the $D^{n}$ separable states residing in the memory just before the crash, corresponding to a mnemonically minimal state, whose basis is usually determined by environment-induced decoherence. The crash causes the system to factor out momentarily from the rest of the universe. The choice of a state in the basis is assumed to be probabilistic, and conforms to the Born rule. The chosen state is 'uploaded' from the core onto the physical system, while memory of all other states is irretrievably erased, as in the crash of a conventional computer program.

As briefly explained in Subsection VIA and to be detailed in Sections VII and VIII macroscopic systems, being computationally unstable, manifest classical behavior, which arises from the very rapid alternating sequence of unitary evolution and information transition. Inasmuch as $\mu=2 n_{\max } \log D$, we infer that the number of particles that can get entangled without suffering information transition ('collapsing') is approximately $\mu$ (apart from a constant factor). Thus, we are again led to the physical interpretation that $\mu$, the degree of fine-graining of Hilbert space, determines quantum/classical border. 
In either scenario presented above, the "Heisenberg cut" 17] that separates the microscopic, quantum mechanical realm from the macroscopic, classical one, is rooted in the information structure of Hilbert space. This is the new insight that emerges in the computational model. On the conceptual side, it furnishes a reasonably good suggestion that physical reality is grounded on informational framework, or, informally, "an information structure lies deeper than physics". Interestingly, this can be interpretted as conveying the converse of Landauer's aphorism: "information is physical" [43]!

How large is $\mu$, or equivalently, $\mathcal{M}_{2}$ ? It must be large enough to permit the well-tested, purely unitary evolution of small systems that are effectively isolated during the course of observations, such as photons in quantum optical experiments and electrons in atoms, that are to good approximation well shielded from environmental influence. Yet $\mu$ is to be small enough that macroscopic systems are computationally unstable, and hence, classical. Here decoherence also plays a role in determining the spatial scales that separate microscopic and macroscopic regimes. An interesting point is that even if $\mathcal{M}_{2}$ is so small that it cannot support the evolution of more than two entangled elementary 2-state objects, it would still suffice to support the separable evolution of all the $\approx 10^{76}$ baryons in the universe, inasmuch as $10^{182 \times 2} \gg 10^{76} 10^{182}$ bits.

We can estimate $\mu$, by examining instances of the largest objects that manifest coherent quantum wavelike properties. The reason is that superposition of macroscopic objects really means entanglement of its constituent objects. Thus, manifestation of wavelike property of a macroscopic body as a whole means that entanglement can be sustained at that level of macroscopicity, as quantified by $n$. Perhaps the largest object (without microscopic degrees of freedom frozen in some way, as in condensates) that have demonstrated wavelike behaviour are fullerene molecules $C_{70} 69$. Similarly, superposition of nucleotides and amino acids have been invoked to explain genetic features in terms of the Grover search algorithm 75]. Let us suppose that the largest, isolated object that can unitarily evolve, without suffering information transition, is an molecule assumed for simplicity to consist homogeneously of $n \mathrm{~N}$ (Nitrogen) atoms. Each $\mathrm{N}$ atom contains $\approx 50$ elementary particles in the form of quarks and electrons. From Eq. (9), we can compute mnemonic entropy as $\mathcal{D}_{\max } \mu=\left(10^{182}\right)^{50 n} \mu=10^{9100 n} \mu$. If we assume that the largest isolated organic molecule that can demonstrate coherent wavelike behavior consists of $1000 \mathrm{~N}$ atoms, then we find $\mathcal{D}_{\max } \mu=10^{9100000} \mu$, so that $\mu=2 \log \left(\mathcal{D}_{\text {max }}\right) \approx 6.0 \times 10^{7}$ bits, and $\mathcal{M}_{2} \approx 6.0 \times 10^{9100007}$ bits $[76]$. This may be compared with the most accurate quantum electrodynamic experiements performed so far, which have about 36-bit (11 decimal places) accuracy, and atomic clocks, which have 33-bit (10 decimal places) accuracy. In fact, the above calculation only considers the degrees of freedom according to known physics, which could well be smaller than the actual memory requirement.

The above numerical estimate for $\mu$ can in principle be updated by looking for larger objects that demonstrate wavelike behavior, either in interferometric set-ups [69, 77] or in complex many-body superpositions [78]. Here it is worth noting that the computational instability must be distinguished from the presence of multiple wavelength scales 76], quantum chaos and decoherence effects. All lead to loss of coherence, albeit for very different reasons. Decoherence affects open systems, but the rest can occur even in closed systems. Decoherence can be excluded by performance of a test experiment in consderable isolation. Careful choice of physical systems and preparation of initial conditions can help thwart quantum chaos [62].

Some points of comparison of the computational model of quantum measurement with exisiting objective indeterministic models thereof can be made. In the dynamical reduction model [34], the state vector reduction is said to be triggered by the spontaneous and entirely random collapses of individual particles in the state (1), which cascades the entire entangled system into a separable state. Comparably, in the present computational model, an information transition is triggered by a segmentation fault in the computational framework supporting physical objects. In the Penrose model [36], the collapse of the wavefunction is attributed to instability of superposition due to gravitational self-energy. Comparably, the present computational model also connects measurement to the a critical phenomenon, namely computational instability. The essential new element in the present model is that the classicality of measurement outcomes and macro-objects is connected with fundamental information processing properties of nature.

\section{Time-scales}

The evolution of physical objects in the above scenario is characterized by two phases, encountered earlier, and two corresponding time-scales: (1) the unitary phase, during which Schrödinger (or its appropriate relativistic generalization) equation strictly applies, and having duration denoted $\tau_{u}$; (2) the transition phase, during which the unitary phase crashes, i.e., the system undergoes information transition, having duration denoted $\tau_{t}$. In typical large macroscopic systems, the rapid cycles of unitary evolution and information transitions give rise to an apparent continuous classical evolution because $\tau_{u}+\tau_{c} \ll 1 \mathrm{sec}$. Neither $\tau_{u}$ nor $\tau_{t}$ is the decoherence time-scale, $t_{\text {decoh }}$, over which long-distance correlations in physical systems practically vanish through purely unitary interactions of a system with its environment.

$\tau_{u}$ can be indefinitely long for isolated, sufficiently small objects, for which no information phase transitions occur, 
since $\mathcal{M} \leq \mathcal{M}_{2}$ is always satisfied. Here, no modifications to Schrödinger dynamics occurs. This is also effectively true in macroscopic systems over length-scales smaller than $\lambda_{d}$, the decoherence length-scale, over which correlations persist inspite of decohering effects due to the environment. To estimate $\tau_{u}$ for systems requiring $\mathcal{M}_{>} \mathcal{M}_{2}$, we begin by defining the information length-scale, $\lambda_{u}$, as the characteristic length-scale of a region whose volume memory support saturates the inequality (17). Therefore, $\mathcal{M}$ for matter within a volume $\lambda_{u}^{3}$ satisfies $\mathcal{M}=\mathcal{M}_{2}$. Thus, $\lambda_{u}$ specifies the lower limit for the length scale above which matter will not be computationally stable. Objects with lengths scale $L$ such that $L \ll \lambda_{u}\left(L \gg \lambda_{u}\right)$ are called microscopic (macroscopic), and will show quantum (classical) behaviour. Therefore, $\lambda_{u}$ spatially marks the "Heisenberg cut" [17], the mesocopic transition from quantum to classical regimes. In a macroscopic body, points separated by distance larger than $\lambda_{u}$ will not be correlated, because coherent effects do not persist over longer length-scales. Since decoherence also produces a similar effect, we conclude that the correlation length scale in a system can not be larger than $\min \left\{\lambda_{d}, \lambda_{u}\right\}$.

Let mean molecular weight $W_{a}$ for the material making up a system be given by $W_{a} \approx 2 N_{a}$, where $N_{a}$ is mean atomic number. Then there are $N_{a}$ electronic and $3 W_{a}=6 N_{a}$ quark, hence a total of $7 N_{a}$, degrees of freedom available per atom. The number of atoms is $n=\rho \lambda_{u}^{3} / W_{a} m_{p}$. It follows from Eqs. (9) and (16) that $182 \times 7 N_{a} n \log 10<\mu / 2$. Solving for $\lambda_{u}$, we obtain:

$$
\lambda_{u}<\left[\frac{\mu m_{p}}{1274(\log 10) \rho}\right]^{1 / 3} \approx 10^{-10}\left[\frac{\mu}{\rho}\right]^{1 / 3} \mathrm{~m}
$$

independent on the atomic specie. Eq. (18) shows that the information length scale becomes smaller at larger densities, in response to the greater compactness of the degrees of freedom available. For a fluid at $\rho=1 \mathrm{gm} / \mathrm{cc}$, with $\mu=10^{12}$ bits, $\lambda_{u}$ is about $10^{-7} \mathrm{~m}$.

We estimate $\tau_{u}$ as the time over which energy can be transmitted across a length $\lambda_{u}$, since this is the period over which entanglement can be set up and memory activated. This implies $\tau_{u}=\lambda_{u} / c_{s}$, where $c_{s}$ is a characteristic speed of energy transport in the body. According to these assumptions, $\tau_{u}$ is independent of size of a macroscopic body. Velocity $c_{s}$ can depend on whether the dominant cohesive energy in the body is in the form of Van der Waals, covalent, ionic or Hydrogen bonds, or if the material is a metal. This suggests that metals, where energy is transmitted most efficiently, by transverse waves set up in the electronic band, are the quickest at becoming classical. In particular, $\tau_{u}=\lambda_{u} / c$. A large metallic body suffers information transitions at the highest frequency. Since it is as yet rather difficult to resolve changes to material properties at such small intervals, sufficiently large bodies behave effectively classically. For a metallic body with $\rho=1 \mathrm{gm} / \mathrm{cc}$, and $\mu=10^{12}$, we found $\lambda_{u} \approx 10^{-7} \mathrm{~m}$. Then $\tau_{u} \geq \lambda_{u} / c=10^{-16}$ sec. For C atoms, which have $12 \times 7=84$ spinorial degrees of freedom, from Eqs. (9) and (16), we obtain $10^{182 \times 84 n}=2^{\mu / 2} \approx 10^{1.5 \times 10^{11}}$. Solving for $n$, we obtain $n \approx 10^{7}$. Therefore, for $\mu=10^{12}$ bits, the largest object made of $\mathrm{C}$ atoms that can in principle manifest coherent quantum behavior, as in the sense of the experiment reported in Ref. [69], is $10^{7} \mathrm{C}$ atoms.

Coincidentally, the expression Eq. (18) can be obtained by estimating $\mathcal{M}_{2}$ in terms of conventional thermodynamic entropy. The temperature that would be obtained if matter density $\rho$ is converted completely to photons can be obtained from the equation for blackbody radiation: $\rho c^{2}=\left(\pi^{2} / 15 \hbar^{3} c^{3}\right)\left(k_{B} T\right)^{4}$. Entropy density is estimated by $S / \lambda_{u}^{3}=4 \rho c^{2} / 3 T$. And we estimate memory support for a volume $\lambda_{u}^{3}$ as $\mathcal{M}=\exp \left(S / k_{B}\right) \mu=$ $\exp \left\{\left(4 \pi^{2} / 45\right)\left(k_{B} T \lambda_{u} / \hbar c\right)^{3}\right\} \mu=\mathcal{M}_{2}$, where $\exp \left(S / k_{B}\right)$ is the total number of states available to the system. Solving for $\lambda_{u}$, we find:

$$
\lambda_{u}<\left[\left(\frac{15}{\pi^{2}}\right)^{1 / 4} \frac{3 \ln \left(\mathcal{M}_{2} / \mu\right)}{4}\right]^{1 / 3}\left[\frac{\hbar}{\rho c}\right]^{1 / 4} \approx 10^{-10}\left[\frac{\mu}{\rho}\right]^{1 / 3} \mathrm{~m}
$$

One way to view the smallness of $\tau_{u}$, is to estimate the spreading of the wavefunction of macroscopic objects during the unitary phase. Consider electrons at temperature $T$, with position uncertainty $\Delta x$ estimated to be their thermal wavelength, i.e., $\Delta x=h / \sqrt{m_{e} k_{B} T} \mathrm{~m}$. Their momentum uncertainty is given by: $\hbar / \Delta x$, so that the subsequent spread of the wavepacket as function of time $t$ is [79]:

$$
\Delta x(t)=\Delta x+\frac{h t}{m_{e} \Delta x},
$$

where $m$ is the mass of the body. The increase in position uncertainty during a unitary phase is $h \tau_{u} / m_{e} \Delta x=$ $\tau_{u} \sqrt{k T / m_{e}}$. For a body with $m=1 \mathrm{gm}$, and at room temperature, assuming $\tau_{u}=10^{-16}$ sec, we find that system can spread through no more than $10^{-11} \mathrm{~m}$. In actual fact, as we later see, the localizing tendency of environmental decoherence will ensure that spreading is much smaller than $\hbar / m \Delta x$, indeed, even negative.

The transition time-scale $\tau_{t}$ should be arbitrarily small, or, more precisely, not larger than $t_{p}$, Planck time, in order to enforce correlations across spacelike-separated measurements on entangled systems [80]. For example, in a 
single particle Young's double slit experiment, detection of the particle at some point on the screen should instantaneously re-set amplitudes at all other points on the screen to zero lest probability conservation be violated. If we interpret the particle's state as information with configuration space as memory address to access that information (probabilistically), then the double-slit experiment requires a nonlocal action on the memory, and we require a speed of information that is arbitrarily large [80, 83]. In the case of entangled systems, the nonlocality (in the sense of violation of Einstein locality [52]) follows also from the violation of Bell's inequality [81], considering that the computational model endows the wavefunction with a subtle, informational realism. Computationally speaking, $\tau_{t}$ is the duration over which nature frees memory and dumps the core across the spacelike region over which the (possibly entangled) wavefunction is spread. As has been recognized, this presupposes a tremendous 'speed of information' 82 ] and a preferred inertial frame [80]. Nevertheless, it can be shown that assumptions of completeness and probablistic nature of measurements suffice to ensure that any model of quantum mechanics does not violate no-signalling and Lorentz-covariance at the level of measurement outcomes even if the speed of information is superluminal [84].

\section{MEASUREMENTS}

The process of measurement, or, more generally, of intervention by an external system in the evolution of system $S$, can be described as taking place in three steps: (a) pre-measurement, in the sense of Eq. (1), whereby measured system $S$ becomes entangled with apparatus $M$; (b) decoherence, which is the loss of phase information via interaction and entanglement of system $S M$ with the environment $Q$ over time $t_{\text {decoh }}$ [13]; (c) a final, least understood, amplification step, where one of the decohered states is obtained as the "classical" outcome (non-selectively described by Eq. (3)).

In the conventional description, the step (c) involves discarding the inaccessible degrees of freedom to obtain a diagonal state in the pointer basis. Why this cannot by itself complete measurement was discussed at some length in the Introduction, namely that it only leads to improper mixtures, which are insufficient to explain selective measurements. The present computational model differs from the above chain of steps only in respect of step (c). Specifically, the information transitions produced via computational instability, introduced in the preceding Section, are shown to act as an engine continually turning improper mixtures to proper mixtures. Thereby we are able to eliminate the main interpretational difficulty that has dogged decoherence as a resolution to the measurement problem, while preserving all its quantitative successes.

The von Neumann pre-measurement in Eq. (10) considers a detector with only one degree of freedom. This would suggest that the required memory support $\mathcal{M} \approx O(\mu D)$, or just $2 \mu\left(\ll \mathcal{M}_{2}\right)$ bits for a qubit, which is insufficient to induce computational instability. A complete description of $M$ and $Q$ involves both macroscopic and microscopic variables. Let us therefore introduce a complete basis for them, namely $\left\{|i, \xi\rangle_{M}\right\}$ and $\left\{|i, \xi, \eta\rangle_{Q}\right\}$, where $i$ labels a macroscopic subspace in $M, \xi$ labels microscopic states in that subspace, and $\eta$ those in $Q$ that tend to correlate with $i, \xi$ in $M$. It is assumed that the microstates are separable, or near separable (localized entanglements that are well screened from macroscopic degrees of freedom are permitted) in the constituent particle states. We note that there need not be the same number of microstates in all the macroscopic subspaces, in $M$ and $Q$, corresponding to a given macroscopic state. We have: ${ }_{M}\left\langle i, \xi \mid i^{\prime}, \xi^{\prime}\right\rangle_{M}=\delta_{i i^{\prime}} \delta_{\xi \xi^{\prime}}$ and ${ }_{Q}\left\langle i, \xi, \eta \mid i^{\prime}, \xi^{\prime}, \eta^{\prime}\right\rangle_{Q}=\delta_{i i^{\prime}} \delta_{\xi \xi^{\prime}} \delta_{\eta \eta^{\prime}}$.

We consider system $S$ in state $\sum_{i} a_{i}|i\rangle_{S}$, where $\left\{|i\rangle_{S}\right\}$ are the eigenstates of the observable that apparatus $M$ is supposed to measure. As an extension of Eq. (11), the pre-measurement step is given by the more general interaction between the quantum system $S$, the apparatus $M$ in 'ready' state $\left|r, \xi_{0}\right\rangle_{M}$ and environment in some state $\left|r, \xi_{0}, \eta_{0}\right\rangle_{Q}$ :

$$
\begin{aligned}
\sum_{i} a_{i}|i\rangle_{S} \otimes\left|r, \xi_{0}\right\rangle_{M} \otimes\left|r, \xi_{0}, \eta_{0}\right\rangle_{Q} \stackrel{H}{\longrightarrow}|\Psi\rangle & =\sum_{i, \xi, \eta} a_{i} \epsilon_{i, \xi}^{M} \epsilon_{i, \xi, \eta}^{Q}|i\rangle_{S} \otimes|i, \xi\rangle_{M} \otimes|i, \xi, \eta\rangle_{Q} \\
& \equiv \sum_{i, \xi, \eta} a_{i}|i\rangle_{S} \otimes|i ; \xi\rangle_{M} \otimes|i ; \xi ; \eta\rangle_{Q}
\end{aligned}
$$

where $\epsilon_{i, \xi}^{M}=\epsilon_{i, \xi}^{M}\left(r, \xi_{0}\right)$ and $\epsilon_{i, \xi, \eta}^{Q}=\epsilon_{i, \xi, \eta}^{Q}\left(r, \xi_{0}, \eta_{0}\right)$ with the terms $r, \xi_{0}, \eta_{0}$ within parenthesis usually omitted for convenience. We have $\sum_{\xi}\left|\epsilon_{i, \xi}^{M}\left(r, \xi_{0}\right)\right|^{2}=1$ and $|i ; \xi\rangle_{M}$ are unnormalized $M$ states whose squared norm gives their statistical weight for given $i$. Similarly, $\sum_{\eta}\left|\epsilon_{i, \xi, \eta}^{Q}\left(r, \xi_{0}, \eta_{0}\right)\right|^{2}=1$ and $|i ; \xi ; \eta\rangle_{Q}$ are unnormalized environmental states whose squared norm gives their statistical weight for given $i$ and $\xi$. The basic idea here is that $M$ amplifies the state of $S$, and $Q$ that of $M$, until, after time $t_{\mathrm{decoh}}$, the entangled environmental states are orthogonal [17], irrespective of microstate labels $\xi_{0}$ and $\eta_{0}$.

Tracing out the environmental degrees of freedom in Eq. (21), we obtain (omitting system subscripts):

$$
\rho_{S M}=\sum_{i, \xi}\left|a_{i}\right|^{2}|i\rangle\langle i|\otimes| i ; \xi\rangle\langle i ; \xi|,
$$


the reduced density operator which possesses the form that we normally associate with non-selective measurements. The recovery of the (block-)diagonalized improper mixture (22) is conventionally thought to complete measurement. While this encounters some interpretational difficulty, nevertheless the fact remains that the dynamical description of subsystems does not depend on whether they are given by of proper or improper mixtures. Such reduced dynamics has been studied by various authors, starting with Lindblad [85] and Gorini, Kossakowski and Sudarshan [86]. In particular, let $\rho$ be the reduced density obtained after environmental degrees of freedom have been traced out. The environment is unknown and assumed to produce random walks of the state vector that are rapid compared to the evolution generated by the system's base Hamiltonian $H_{0}$. Thus $\rho$ undergoes a Brownian motion superposed on an ideal motion, the result of which can be shown to follow the Lindblad equation:

$$
\hbar \frac{\partial \rho}{\partial t}=i\left[H_{0}, \rho\right]-\frac{1}{2} \sum_{k}\left(\hat{L}_{k}^{\dagger} \hat{L}_{k} \rho+\rho \hat{L}_{k}^{\dagger} \hat{L}_{k}-2 \hat{L}_{k}^{\dagger} \rho \hat{L}_{k}\right)
$$

with arbitrary generators $\hat{L}_{k}$ in $\mathcal{H}$ [85]. These observations, and the main conclusions that follow below, are not affected by weakening some of the assumptions that go into the model of measurement given above: for example, the states of $M$ and $Q$ can in general be impure, and $Q$ states need not be coupled to $M$ 's microstates.

The Bassi-Ghirardi theorem [8] implies that von Neumann's chain of efficient causes in the course of measurement cannot be terminated without departure from linear superposition, unless we admit a many-worlds interpretation [17]. In the computational model, this chain is terminated at finite depth, when sufficient entanglement has accumulated in the $S M Q$ system so that $\mathcal{M}=\mathcal{M}_{2}$. When the $\mathcal{M}_{2}$ bound is exceeded, the physical system becomes computationally unstable, and information transitions into a proper mixture. Thus, the main theme here is that: Information transitions induced by computational instability continually drive (mixtures of) improper mixtures into (mixtures of) proper mixtures. Often, the effect of information transitions can be treated as a continuous process, even though they are jerky, unlike decoherence. Let us estimate $\mathcal{M}$ induced by a $D$-dimensional obervable $\hat{A}$ of $S$. The pointer on $M$, assumed to be made of silicon, must have $D$ macroscopically distinguishable positions. Suppose the pointer weighs $1 \mathrm{gm}$, and thus has about $n=3.5 \times 10^{22} \mathrm{Si}$ atoms, or $7 n=2.5 \times 10^{23}$ spinor degrees of freedom. According to Eq. (9), an estimate for memory is $\mathcal{M}(S M)=D \mathcal{D} \mu=D \times 10^{182 \times 7 n} \mu \approx 10^{3.0 \times 10^{26}} D \mu$ bits. Clearly, even though possibly

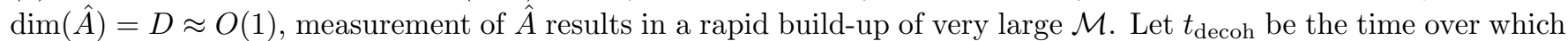
a system decoheres, i.e., over which $\rho_{S M}$ becomes spatially block-diagonalized on account of interaction with the environment. Suppose $\tau_{u} \geq t_{\text {decoh. }}$. This implies that $\mathcal{M}$ is sufficiently large, so that the information transitions come after environmental decoherence in the observational chain of events. (Later we shall find that the main conclusions follow even if $\left.\tau_{u}<t_{\text {decoh }}\right)$.

With these assumptions, consider the system $S$ at $t=0$ in the initial state in (21), when it is brought in contact with $M$ for measurement. As the atoms on $S$ interact with those in $M$, and the latter with atoms in $Q$, the resultant entangling effect propagates into the environment until, at $t=\tau_{u}$, because $\mathcal{M}=\mathcal{M}_{2}$, there is a memory overflow in the combined system $S M Q$. Consequently, it becomes computationally unstable and suffers an information transition ('a crash'), whereby of the larger than $10^{\left(3 \times 10^{26}\right)} D$ entangled possibilities, a single random state $|i\rangle|i, \xi\rangle|i, \xi, \eta\rangle$ is selected, with probability $\left|a_{i} \epsilon_{i, \xi}^{M} \epsilon_{i, \xi, \eta}^{Q}\right|^{2}$, with information of all other states being irreversibly erased from the system's memory. Memory thus freed is now available for further Schrödinger evolution in the succeding unitary phase. The probability that outcome $i$ is obtained on the measured system $S$ is $\left|a_{i}\right|^{2}=\sum_{\xi, \eta}\left|a_{i} \epsilon_{i, \xi}^{M} \epsilon_{i, \xi, \eta}^{Q}\right|^{2}$. Thus, we recover the usual Born rule for projection. Thereby, non-selectively speaking, information transition results in a proper ensemble of $S$ states correlated with the pointer basis elements of $M$, starting from the improper mixture of $S M$ at the end of the preceding unitary phase. The particular state resulting from the information transition serves as initial condition for the next unitary phase that continues briefly until the next transition phase. The evolution of macroscopic systems consists of such repeated cycles of unitary and transition phases.

Considering that measurement outcomes are non-superpositional in the pointer-basis, the duration $\tau_{u}$ of the unitary phase cannot be larger than the time over which the value of the measured observable of $S$ is read out from $M$, i.e., $\tau_{u}<t_{\text {read:out. }}$ Typically, $t_{\text {read:out }} \leq 10^{-3}$ sec. Therefore, $\lambda_{u}$ can in principle be as large as $10^{-3} c=10^{5} \mathrm{~m}$, so that according to Eq. (18),$\mu>10^{45}$ bits. We note that although the $S M Q$ system could have decohered much earlier, only the final amplified read-out guarantees that the $S M$ mixture is proper, i.e., the particular read-out state is pure. It is important to note that, the mechanism of decoherence assures us that, post-measurement, the pointer position at some $i$ is robust because, under fluctuations due to quantum uncertainty or more conventional environmental action (namely, noise), it migrates only over $\xi$ in a subspace $\{|i, \xi\rangle\}$ labelled by fixed $i$ [17]. The pointer state is therefore only passively recognized by the environment. Indeed, a measuring device would be useless if its pointer states were not stable against decoherence.

Non-selectively, one can view the state $\rho_{S M Q}^{\prime}$ of the system just after information transition as obtained as if the environment locally 'measures' the state $\rho_{S M Q} \equiv|\Psi\rangle\langle\Psi|$ in the $\{|i\rangle|i, \xi\rangle|i, \xi, \eta\rangle\}$ basis. Here $|\Psi\rangle$ is given by Eq. (21). We call this basis in $Q$, which determines the ensemble into which the system collapses upon undergoing 
information transition, as the 'transition basis'. 'Measurement' in this leads to the 'transition ensemble', denoted $\mathcal{E}_{1} \equiv\left\{\left|a_{i}\right|^{2}|i\rangle|i ; \xi\rangle|i ; \xi ; \eta\rangle\right\}$, whose density operator is given by the block-diagonal matrix:

$$
\rho_{S M Q}^{\prime}=\sum_{i, \xi, \eta}\left|a_{i}\right|^{2}|i\rangle\langle i|\otimes| i ; \xi\rangle\langle i ; \xi|\otimes| i ; \xi ; \eta\rangle\langle i ; \xi ; \eta| .
$$

In this juncture, the state of $\rho_{S M Q}^{\prime}$ momentarily carves out into a separable state from the rest of the universe. Therefore, non-selectively speaking, the system $S M Q$, and indeed the universe, even as a whole, now exist in a mixed state. In contrast, in an entirely unitary evolution scenario, the final state of $S M Q$ remains a pure, entangled state of the form in Eq. (21).

Inspite of this, it is easy to see that the computational model implies the same reduced dynamics as unitary Schrödinger evolution. This follows from the result that the reduced density operator of a subsystem is unaffected by local operations on another subsystem [5, 71]. In particular, the dynamics of the $S M$ subsystem is independent of 'measurements' and the choice of the transition basis in $Q$, i.e.,

$$
\operatorname{Tr}_{Q}(|\Psi\rangle\langle\Psi|)=\operatorname{Tr}_{Q}\left(\rho_{S M Q}^{\prime}\right) .
$$

This means the master equation for the subsystem $S M$ is the same, whether or not the system as a whole is subjected to information transitions. In general, this implies that the evolution of $\rho$, the reduced density matrix for a subsystem not isolated from the environment, is governed by the Lindblad equation (23) even in the computational model. The new element lies in the interpretation: $\rho$ in the conventional description is an improper mixture of states, whereas $\rho$ is a proper mixture of pure states in the computational model. As a result, in the latter model there arises no difficulty in reconciling the observed purity of the final outcome on $S$ in a given individual measurement with the final state obtained according to the formalism. The model thus resolves the measurement problem.

In the context of the famous Schrödinger cat paradox [9], we can regard the hapless cat, or more precisely the state of feline life or death, as pointer states to some quantum (emission) process. The act of entangling the process with the cat generates an immense entanglement. The resulting computational instablity causes the initial coherent superposition of the process to become a proper incoherent mixture, classically correlated with the cat's state.

Measurement in a purely unitary dynamics can in principle be reversed by suitably accessing environmental degrees of freedom, and in general those of the entire universe. In the computational model, they are in principle irreversible. As these degrees of freedom are fairly inaccessible, and most realistic measured systems are open, the computational model is practically indistinguishable from standard quantum theory in this respect. All the essential above arguments for the resolution of the measurement problem in the computational model hold good even if $\tau_{u}<t_{\mathrm{decoh}}$, since reduced dynamics is independent of the propriety (status of being proper or improper) of the mixture. However, as shown below, only at $t>t_{\text {decoh }}$ does a preferred transition basis for the environment, determined by decoherence, emerge.

The discussion above presumes that the transition ensemble of states $\{|i\rangle|i, \xi\rangle|i, \xi, \eta\rangle\}$ is determined by premeasurement and decoherence, given by (21). This implies that the triple basis in the $S M Q$ system, comprising of $\left\{|i\rangle_{S}\right\}$ (the eigenstates of the measured observable $\hat{A}$ ), $\left\{|i, \xi\rangle_{M}\right\}$ (the pointer (micro-)states), and $\left\{|i, \xi, \eta\rangle_{Q}\right\}$ (the environmental eigenstates correlated with $M$ states) is the preferred basis for information transition, reflecting the well-known "(preferred) basis problem" [72]: that measurement leaves the system as definite in a preferred basis. We denote this basis for $\mathcal{H}_{S} \otimes \mathcal{H}_{M} \otimes \mathcal{H}_{Q}$, correlated with $M$ 's pointer states, as the 'extended pointer basis'. The measurement problem can then be posed thus: why is the extended pointer basis superselected to be the transition basis? The answer arises naturally in the computational model. Since the system's information transition occurs because of memory overflow, it is reasonable that the ensemble it leads to should on average require minimal memory support. In the above example, the reduction in memory support from state (21) to some random separable state $|i\rangle|i, \xi\rangle|i, \xi, \eta\rangle$ is exponential- to be precise, $\mathcal{D}$-fold. Now, if the system is completely decohered, the states $|i, \xi, \eta\rangle_{Q}$ will be almost orthogonal to each other, and the transition mixture will contain only states separable in the tripartite system $S M Q$, namely elements from the extended pointer basis. Let $D \equiv \operatorname{dim}\left(\mathcal{H}_{S}\right)$, and $M$ be constituted of $\bar{m}$ elementary particles, $Q$ of $\bar{q}$ elementary particles. Then, the memory support required for the right hand side state in Eq. (21) is $\mathcal{M}(|\Psi\rangle)=D 10^{182(\bar{m}+\bar{q})} \mu$. In ensemble $\mathcal{E}_{1}, S$ and $M$ (and its constituents, given the microscopic correlations) exist in separable states, so that memory support required for any state in $\mathcal{E}_{1}$ is $\mathcal{M}\left(\mathcal{E}_{1}\right)=\left(D+(\bar{m}+\bar{q}) 10^{182}\right) \mu \ll \mathcal{M}(|\Psi\rangle)$. Here it is worth recollecting that $\mathcal{M}$ is an absolute quantity. The exponentially large difference between $\mathcal{M}\left(\mathcal{E}_{1}\right)$ and $\mathcal{M}(|\Psi\rangle)$ holds inspite of Eq. 25.

An information transition into any other basis leads to entanglement in the transition ensemble, and hence on average larger memory requirement. Suppose the transition basis is $\left|i^{\prime}, \xi^{\prime}, \eta^{\prime}\right\rangle_{Q} \equiv \sum_{i, \xi, \eta} U_{i^{\prime} \xi^{\prime} \eta^{\prime}}^{i \xi \eta}|i ; \xi ; \eta\rangle_{Q}$, where $U_{i^{\prime} \xi^{\prime} \eta^{\prime}}^{i \xi \eta}$ is a unitary transformation in $\mathcal{H}_{Q}$, satisfying $\sum_{i, \xi, \eta} U_{i^{\prime} \xi^{\prime} \eta^{\prime}}^{i \xi \eta} U_{j^{\prime} \phi^{\prime} \gamma^{\prime}}^{i \xi \eta}=\delta_{i^{\prime} j^{\prime}} \delta_{\xi^{\prime} \phi^{\prime}} \delta_{\eta^{\prime} \gamma^{\prime}}$. The eigenstates $\left\{\left|i^{\prime}, \xi^{\prime}, \eta^{\prime}\right\rangle_{Q}\right\}$ are not correlated with the pointer basis of $M$. At the end of the pre-measurement stage, we can rewrite the final state 
of Eq. (21) as:

$$
\sum_{i^{\prime}, \xi^{\prime}, \eta^{\prime}}\left(\sum_{i, \xi, \eta} U_{i^{\prime} \xi^{\prime} \eta^{\prime}}^{i \xi \eta} a_{i}|i\rangle_{S} \otimes|i ; \xi\rangle_{M}\right) \otimes\left|i^{\prime} ; \xi^{\prime} ; \eta^{\prime}\right\rangle_{Q}
$$

The ensemble (of unnormalized states) that would result from an information transition wherein $Q$ 'measures' in the primed basis is denoted $\mathcal{E}_{2} \equiv\left\{\left(\sum_{i, \xi} \alpha_{i, \xi}\left(i^{\prime} \xi^{\prime} \eta^{\prime}\right) a_{i}|i\rangle_{S} \otimes|i ; \xi\rangle_{M}\right) \otimes\left|i^{\prime} ; \xi^{\prime} ; \eta^{\prime}\right\rangle_{Q}\right\}$, where $\alpha_{i, \xi}\left(i^{\prime} \xi^{\prime} \eta^{\prime}\right) \equiv \sum_{\eta} U_{i^{\prime} \xi^{\prime} \eta^{\prime}}^{i \xi \eta}$ We note that $\mathcal{E}_{2}$ is an ensemble of $S M$ entangled states classically correlated with $Q$ states. As the density operators corresponding to the two ensembles are identical, i.e., $\rho\left(\mathcal{E}_{1}\right)=\rho\left(\mathcal{E}_{2}\right)$, the two ensembles are locally indistinguishable, and quantified by the same amount of von Neumann information and of quantum entanglement, as quantified using any suitable generalization of conventional methods applicable to pure states 73 .

In contrast, state information $\mathcal{M}$, being an absolute informational measure, depends on the ensemble of states, rather than on the density matrix. In ensemble $\mathcal{E}_{2}$, the system $S M$ exists in an entangled states with very large Schmidt ranks. On the other hand, $Q$ exists in a definite microstate, with its constituents in a separable state: $\mathcal{M}\left(\mathcal{E}_{2}\right)=D \times 10^{182 \bar{m}} \times \bar{q} 10^{182} \mu=D \bar{q} 10^{182(\bar{m}+1)} \mu \gg \mathcal{M}\left(\mathcal{E}_{1}\right)$. Thus, ensemble $\mathcal{E}_{2}$ is not mnemonically minimal even though $\mathcal{M}\left(\mathcal{E}_{2}\right) \ll \mathcal{M}(\Psi)$. As the information transition aims to free the segmentation faulted memory supporting a physical system, the transition basis and transition ensemble that minimize state information will be preferred. In particular, the mnemonically minimal mixture $\mathcal{E}_{1}$, that corresponds to the extended pointer basis in (21), is the preferred outcome of information transition. Any other basis, and the resultant ensemble $\mathcal{E}_{2}$ is dispreferred as it does not enable the largest freeing of memory. This can be stated as a fundamental superselection principle ('mnemonic minimization principle'): the transition-basis is given by one that minimizes state information. Therefore, decoherence helps select the preferred basis to play the role of the transition-basis for the $S M Q$ system, in this case the extended pointer basis. Information transitions play the complementary role of converting improper mixtures to proper mixtures in this selected basis. Thus, in conjunction with decoherence, the computational model resolves the basis problem.

Actually, in the above measurement process, the role of the decohering environment is sufficient, but not necessary, to explain measurement. Merely the assumption $\left\langle i, \xi \mid i^{\prime}, \xi^{\prime}\right\rangle=\delta_{i i^{\prime}} \delta_{\xi \xi^{\prime}}$ and the mnemonical minimization principle suffice to obtain the mixture Eq. (22). With these assumptions, the role of $Q$ is not very different from the role of $M$. However, we include $Q$ because in fact interaction with the environment is inevitable. Furthermore, environmental decoherence plays another important role: it ensures that the extended pointer basis indeed consists of localized wavepackets 10]. As a result, the states in the transition ensemble also correspond to localized wavepackets, which is essential to explain why measuring apparatuses and their pointers manifest particle-like localization. Decoherence in the present model thus plays the twin important roles of (1) helping determine the transition-basis; (2) localization of decohered states.

If $\tau_{u}<t_{\text {decoh }}$, the system has not entirely decohered during early information transitions, and the environmental states correlated with the $|i\rangle|i, \xi\rangle$ 's in Eq. (21) are not the mutually orthogonal vectors $\{|i, \xi, \eta\rangle\}$, but a set of non-orthogonal vectors, which we denote by $\{\mid \underline{i} ; \xi ; \eta \eta\}$. The mnemonic minimization principle still implies a unique transition ensemble: $\mathcal{E}_{1}^{\prime} \equiv\left\{\left|a_{i}\right||i\rangle|i ; \xi\rangle|\underline{i} ; \xi ; \eta\rangle\right\}$, with no preferred environmental basis. Ensemble $\mathcal{E}_{1}^{\prime}$ may be considered as being obtained when an incompletely decohered version of state $|\Psi\rangle$ in Eq. (21) is 'measured' by $M$ in the $\{|i, \xi\rangle\}$ basis. Hence, in place of $\rho_{S M Q}^{\prime}$ in Eq. (24), we get:

$$
\rho_{S M Q}^{\prime \prime}=\sum_{i, \xi, \eta}\left|a_{i}\right||i\rangle\langle i|\otimes| i ; \xi\rangle\langle i ; \xi|\otimes| \underline{i} ; \underline{\xi} ; \underline{\eta}\rangle\langle\underline{i} ; \underline{\xi} ; \underline{\eta}| .
$$

This makes little difference to measurement observer because $\operatorname{Tr}_{Q}\left(\rho_{S M Q}^{\prime \prime}\right)=\operatorname{Tr}_{Q}\left(\rho_{S M Q}^{\prime}\right)=\operatorname{Tr}_{Q}(|\Psi\rangle\langle\Psi|)$. Once the system fully decoheres, which is quite rapid [13], the extended pointer basis, built up from localized particles, emerges as the preferred basis. In particular, considering that pointers are localized and the read-out is classical, we expect that $t_{\text {read:out }}$, the minimum time required for $M$ 's measurement result to be read out, $\operatorname{satisfies} t_{\text {read:out }}>\max \left(\tau_{u}, t_{\text {decoh }}\right)$. Thus, together with decoherence, the computational model resolves the measurement problem and the basis problem in a way that agrees with classical experience and quantum theory as applied to well-tested quantum phenomena.

In constrast to almost all other models for quantum measurement (save perhaps the many-worlds interpretation), the present computational model can be said to introduce minimal new physics, according to two criteria. One is that, the computational model is identical with Schrödinger evolution for microscopic systems, and implies the same subsystem dynamics as Schrödinger evolution for open systems. Microscopic systems include isolated objects like photons in space or in quantum optical set-ups. Subatomic particles like electrons in atoms that are well screened from getting strongly entangled with macroscopic degrees of freedom and whose potentials have length-scales much smaller than $\min \left\{\lambda_{d}, \lambda_{u}\right\}$ are also effectively unaffected by information transitions and behave quantum mechanically. Open systems include most macroscopic systems, such as measuring apparatuses. Thus, the computational model is 
identical/consistent with quantum dynamics in all the relevant and accessible regimes in which quantum mechanics has been tested. The second reason is that information transitions, which essentially distinguish the computational model from standard Schrödinger evolution, are formally like familiar measurements. Moreover, information transitions are not dynamical processes, in the sense following sense. As pointed out in Section VIC the transition in Eq. (21) is almost instantaneous across configuration space. It cannot be described dynamically within the spacetime arena, except possibly in a pre-quantum theory for the computational architecture underlying information transitions, which is beyond the scope of the present work. We believe information transitions are best understood as such: as computational procedures, that represent a window onto nature's basic information theoretic framework.

One point worth noting is that it is not just the size of the objects involved, but the number of degrees of freedom becoming available during interaction, that determines whether or not the interaction will lead to information transitions. This may mean, for example, that certain internal degrees of freedom may be separable or contain sufficiently low energy, that they may be irrelevant to compute $\mathcal{M}$. A simple illustration of this is found in the fact that it is absorption rather than deflection or rotation (of the polarization of) of photons that plays a more important role in engendering information transition in optical systems. Indeed, a massive mirror or lens preserves superpositions but a small detector does not. The latter is the stronger 'state reductor'. It is not simply the mass of the optical element, but also the operation, that counts. In reflection or refraction, an electron excited by the incoming photon, or the atom to which the electron transmits the photon, re-transmits the photon elastically. As a result, the passage of the photon does not create entanglement among electrons and atoms along its path. Non-absorptive photon manipulations do not consume much memory, and do not lead to information transitions. They preserve the coherence of the incoming beam. In contrast, absorption leads to an unpredictable scattering, excitation and interaction of particles along the photon's path, which leads to large entanglement and thence information transition. As a result, even a tiny photon-detector is a more efficient state reductor than a massive mirror. A rigorous study of the photon's scattering and its absorption, and thence the correct estimate for $\mathcal{M}$, should take into consideration the many possible configurations in which heat can be dissipated through and from a detector. Now, scattering is also known to be an efficient decoherer [10, 17]. We thus obtain the thumb rule that processes that increase entropy and lead to strong decoherence are also efficient at inducing information transitions.

\section{EMERGENCE OF CLASSICALITY IN THE MACROSCOPIC WORLD}

As in other models of measurement, classicality of the macroscopic world is closely related to the fact that outcomes of quantum measurements are classical. Closed, small systems, for which $\mathcal{M}<\mathcal{M}_{2}$ or whose size $L$ satisfies $L<\lambda_{u}$, are entirely quantum mechanical. Their dynamics is captured by Schrödinger equation, or the appropriate relativistic generalizations thereof.

On the other hand, a macroscopic body, for which $L>\lambda_{u}$, will evolve naturally into a highly entangled system in terms of its basic degrees of freedom, leading to computational instability. The resultant rapid cycles of information transition and unitary evolution over a timescale of $\tau_{u}$ cause it to behave classically. Its state is described by a proper mixture or, alternatively, probablistic evolution of a pure state. We note that, even in macroscopic bodies, subatomic particles like photons in quantum optical experiments, and electrons in atoms, that are well screened from macroscopic degrees of freedom and whose potential varies significantly over distance $\min \left\{\lambda_{d}, \lambda_{u}\right\}$ are also effectively unaffected by information transitions and hence behave quantum mechanically. Macroscopic systems $Z$ that we usually encounter are open systems, decohered by environment $Q$, which leads to to the localization of its constituent particles. A macroscopic open system is thus described as a proper mixture of localized particle states. We will use this insight to see why Schrödinger cat states (macroscopic systems existing in coherent superpositions of macroscopically distinguishable states) are forbidden.

The correct quantitative way to determine whether a system is computationally unstable will depend on the ultimate constitution, and hence true degrees of freedom, of matter. However, we can hope to get approximately correct answers if we compute $\mathcal{M}$ and $\mathcal{M}_{2}$ using the same model for the microscopic constitution of matter. In so far as the chosen model of material constitution is not basic, both $\mathcal{M}$ and $\mathcal{M}_{2}$ will be similarly underestimated.

It is well acknowledged that a macroscopic object like a cat does not exist in a Schrödinger cat [9] state like:

$$
\left.\left.|v\rangle=\frac{1}{\sqrt{2}}(\mid \text { here }\rangle+\mid \text { there }\right\rangle\right) .
$$

As in the preceding Section, we introduce the various microstates $\xi$ into the macroscopically distinguishable states of spatial proximality and distality, namely, $\{\mid$ here,$\xi\rangle, \mid$ there, $\xi\rangle\}$, satisfying $\langle$ here, $\xi|$ here, $\left.\xi^{\prime}\right\rangle=\langle$ there, $\xi|$ there, $\left.\xi^{\prime}\right\rangle=\delta_{\xi \xi^{\prime}}$, and $\langle$ here, $\xi|$ there, $\left.\xi^{\prime}\right\rangle=0$. Over a period of time $t_{\text {decoh }}$, the environmental states the cat is entangled with become orthogonal states $\{\mid$ here, $\xi, \eta\rangle$, |there, $\xi, \eta\rangle\}$ satisfying $\langle$ here, $\xi, \eta|$ here, $\left.\xi^{\prime}, \eta^{\prime}\right\rangle=\langle$ there, $\xi, \eta|$ there, $\left.\xi^{\prime}, \eta^{\prime}\right\rangle=\delta_{\xi \xi^{\prime}} \delta_{\eta \eta^{\prime}}$, and 
$\langle$ here, $\xi, \eta|$ there, $\left.\xi^{\prime}, \eta^{\prime}\right\rangle=0$. With these definitions, in place of Eq. (28) we have for the state of the combined cat+environment system:

$$
\left.\left.\left.\left.|\Upsilon\rangle=\sum_{\xi, \eta} \frac{1}{\sqrt{2}}\left(\alpha_{\xi, \eta} \mid \text { here, } \xi\right\rangle \mid \text { here, } \xi, \eta\right\rangle+\beta_{\xi, \eta} \mid \text { there, } \xi\right\rangle \mid \text { there, } \xi, \eta\right\rangle\right)
$$

where $\sum_{\xi, \eta}\left|\alpha_{\xi, \eta}\right|^{2}=\sum_{\xi, \eta}\left|\beta_{\xi, \eta}\right|^{2}=1$. We estimate that a $1.4 \mathrm{~kg}$ cat contains about $n=1.4 / 14 m_{p} \approx 10^{26} \mathrm{~N}$ atoms (on average), or $7 n$ basic, quark and electronic degrees of freedom. This corresponds to $\mathcal{M}_{\text {cat }} \approx\left(10^{182}\right)^{7 n} \mu=10^{1.2 \times 10^{29}} \mu$ bits to represent the cat alone. The total mnemonic support $\mathcal{M}_{\text {tot }}$ for the cat+environment system is given by $\log \mathcal{M}_{\text {tot }} \approx \log \mathcal{M}_{\text {cat }}+\log \mathcal{M}_{\text {env }}$, where $\mathcal{M}_{\text {env }}$ is the memory needed for the unknown (but immense) environment. It is reasonable to expect that $\mathcal{M}_{\text {env }} \gg \mathcal{M}_{\text {cat }}$.

From the observed classicality of cats, it follows that the state $|\Upsilon\rangle$ in Eq. (29) requires $\mathcal{M}>\mathcal{M}_{2}$ bits to specify. Such a state, being computationally unstable, cannot survive for much longer than $\tau_{u}$ sec, after which time it suffers an information transition. It repeatedly undergoes such transitions that interrupt unitary phases lasting about $\tau_{u}$ sec. After time $t \geq t_{\mathrm{decoh}}$, decoherence superselects a preferred cat+environment basis, consisting of localized wavepackets, given by the basis in which Eq. (29) is written, which emerges as the transition basis. Loosely, one could say that in a given episode of information transition, the environment 'measures' the state $|\Upsilon\rangle$ in the basis $\{\mid$ here, $\xi, \eta\rangle, \mid$ there, $\xi, \eta\rangle\}$, information-transitioning the cat into a specific microscopic state of the cat, say a $\mid$ here, $\xi\rangle$ with probability $\sum_{\eta}\left|\alpha_{\xi, \eta}\right|^{2} / 2 \equiv\left|\alpha_{\xi}\right|^{2} / 2$, or a $\mid$ there, $\left.\xi\right\rangle$ with probability $\sum_{\eta}\left|\beta_{\xi, \eta}\right|^{2} / 2 \equiv\left|\beta_{\xi}\right|^{2} / 2$. Nonselectively speaking, the cat is transformed from state (29) into one of the states in the transition ensemble $\mathcal{E}_{1}=$ $\{\mid$ here; $\xi\rangle, \mid$ there; $\left.\left.\xi^{\prime}\right\rangle\right\}$ given by the proper mixture:

$$
\begin{aligned}
\rho_{\text {cat }} & \left.\left.=\frac{1}{2} \sum_{\xi}\left(\left|\alpha_{\xi}\right|^{2} \mid \text { here, } \xi\right\rangle\left\langle\text { here, }\left.\xi|+| \beta_{\xi}\right|^{2}\right| \text { there, } \xi\right\rangle\langle\text { there, } \xi|\right) \\
& \left.\equiv \frac{1}{2} \sum_{\xi}(\mid \text { here } ;\rangle\langle\text { here } \xi \xi|+| \text { there } \xi\rangle\langle\text { there } \xi \xi|\right) .
\end{aligned}
$$

This entails the interpretation that the cat actually exists in one of the pure states that enter into the mixture (301). Decoherence ensures that the macroscopically distinguishable states of $\mid$ here $\rangle$ or $\mid$ there $\rangle$ are robust and made up of localized particles. After a given information transition, the cat evolves only into superpositions of microstates corresponding to one of these macrostates, before being reduced into one of those microstates by the succeeding information transition, and so on. In this way, the familiar classical cat emerges as the combined effect of decoherence and information transitions.

The effect of large numbers of interacting degrees of freedom on the decay of macroscopic superposition 74] is central to the present model as it is to most models of measurement. We can summarize this line of argument for the classicality of the macroscopic world as follows: macroscopic superposition $\longrightarrow$ very large entanglement involving microscopic degrees of freedom $\longrightarrow$ computational instability $\longrightarrow$ classical regime via rapid cycle of information transitions and unitary evolution.

As in the case of measurement, the environment is not needed for "classicalization" in the sense that for a sufficiently large cat, it may be true that $\mathcal{M}_{\text {cat }}>\mathcal{M}_{2}$. However, interaction with the environment is inevitable in most usual cases, and environmental decoherence helps explain localization of particles. As shown by Joos and Zeh [10], decoherence due to scattering processes frequently causes this effect by means of unitary destruction of coherence between macroscopically separated positions. Consider the scattering of an object in state $|\chi\rangle$ off a particle in state $\int_{x} \phi(x)|x\rangle d^{3} x:$

$$
\int_{x} d^{3} x \phi(x)|x\rangle|\chi\rangle \stackrel{t}{\longrightarrow} \int_{x} d^{3} x \phi(x)|x\rangle S_{x}|\chi\rangle
$$

where the scattered state $S_{x}|\chi\rangle$ may be calculated using an appropriate S-matrix. The reduced density operator for the scatterer changes as $\phi(x) \phi^{*}\left(x^{\prime}\right) \longrightarrow \phi(x) \phi^{*}\left(x^{\prime}\right)\left\langle\chi\left|S_{x^{\prime}}^{\dagger} S_{x}\right| \chi\right\rangle$. A single scattering like this cannot quench the offdiagonal terms. Let $k, F, \sigma_{\text {eff }}$ be, respectively, the wave-number, flux and total cross section of the incoming particles. Letting $\Lambda \equiv k^{2} F \sigma_{\text {eff }}$, one finds:

$$
\rho\left(x, x^{\prime}, t\right)=\rho\left(x, x^{\prime}, 0\right) \exp \left\{-\Lambda t\left(x-x^{\prime}\right)^{2}\right\}
$$

implying an exponentially rapid dying off of the off-diagonal terms [10].

Combining the damping in Eq. (32) due to decoherence with "free" Schrödinger evolution, we obtain:

$$
\frac{\partial \rho\left(x ; x^{\prime} ; t\right)}{\partial t}=\frac{i}{2 m}\left(\frac{\partial^{2}}{\partial x^{2}}-\frac{\partial^{2}}{\partial x^{\prime 2}}\right) \rho-\Lambda\left(x-x^{\prime}\right)^{2} \rho
$$


where, the first term in right hand side represents the free evolution with $H_{0}=p^{2} / 2 m$, in coordinate representation, in the Lindblad equation Eq. (23). One obtains the non-Hamiltonian part in Eq. (33) as a special case of the Lindblad equation, setting $\hat{L}_{k}^{\dagger}=\hat{L}_{k}$. In this case, the Lindblad terms can be re-written as $\hat{L}^{2} \rho+\rho \hat{L}^{2}-2 \hat{L} \rho \hat{L}=[\hat{L},[\hat{L}, \rho]]$. For $\hat{L}=\sqrt{2 \Lambda} \hat{x}$, one recovers Eq. (33).

In practice, the localizing tendency in Eq. (32) is to be balanced against the natural spreading tendency of wavepackets. This suggests that there is a finite scale, which we call the decoherence length-scale $\lambda_{d}$, representing the 'equilibrium' size of wavepackets. Because coherence is more or less preserved at length-scales smaller than $\lambda_{d}$, phenomena confined to such small scales are effectively unaffected by information transitions, and behave quantum mechanically even in a macroscopic body, provided $\lambda_{d}<\lambda_{u}$. Otherwise, the relevant quantity is the minimum of the two length-scales. Microscopic objects such as electrons in atoms or photons in a conventional quantum optical experiment will move through such structures according to the rules of quantum mechanics, rather than classical mechanics, if the length scale of the potentials they are responding to is smaller than $\min \left\{\lambda_{d}, \lambda_{u}\right\}$, which scale can, from this viewpoint, be regarded as the border scale between microscopic and macroscopic phenomena.

It is worth noting that macroscopic matter is not necessarily classical. As is well known, quantum behaviour has been noted in macroscopic systems manifesting coherent matter states like Bose-Einstein condensates (BECs) [87] and in Schrödinger cat states observed in superconducting quantum interference devices (SQUIDs) [88]. In such systems, the microscopic degrees of freedom are frozen, as a result of which $\mathcal{M} \ll \mathcal{M}_{2}$. Indeed, whereas $n$-partite entanglement requires exponentially more memory support than for $n$ particles in separable states, BECs, superconductors or some other such coherent state matter, require less. From this standpoint, condensation is the opposite of entanglement. A condensate has almost zero entropy in that all particles collectively occupy the same single many-particle ground state. For example, the Schrödinger cat states observed in SQUID superconductors are described by macroscopic clockwisecounterclockwise oscillations of the flux through a loop, a collective coordinate representing the motion of $\sim 10^{9}$ Cooper pairs acting in tandem. Since the experimental temperature is 1000 times smaller than the superconducting energy gap, almost most microscopic degress of freedom are frozen out and only the collective flux coordinate retains any dynamical relevance [88].

Let us consider a system of $n$ elementary 2-state objects restricted to volume $\mathcal{V}$. As seen earlier, state information for the system if the particles are non-interacting, and thus their states remain separable, is $\mathcal{M}_{\text {separable }}=n 10^{182} \mu$. In the case of ordinary matter, where interaction causes entanglement, $\mathcal{M}_{\text {usual }}=10^{182 n} \mu$. Suppose the system is a condensate. As its coherent matter involves elementary objects (atoms or Cooper pairs of electrons) 'coalescing' into and behaving like a single, coherent, non-interacting whole, the required memory is $\mathcal{M}_{\text {coherent }}=10^{182} \mu \ll \mathcal{M}_{2}$. Considering that we estimated $\mathcal{M}_{2}$ to be sufficiently large as to support even the separable evolution of all particles in the universe, it follows that coherent matter will not suffer information transitions at all, and can be treated as entirely quantum mechanical.

\section{CONCLUSIONS}

The information processing involved in the evolution of physical systems, viewed as computations performed by nature, were quantified using the physics of information processing. The role of entanglement in exponentially augmenting its requirement was pointed out. In particular, in a system with $n$ entangled subsystems of average energy $E$ and identically of dimension $D$, computational speed requirement goes as $\sim 2^{\mu / 2} D^{n} n E$ op/s, whilst memory allocation requirement goes as $\sim D^{n} \mu$. However, most of this is physically inaccessible, essentially because of Heisenberg uncertainty, except when suitably designed interference manifests it, as in a Young's double-slit experiment or Shor's prime factorization algorithm 53]. Why is only a fraction of the underlying state information accessible for classical information processing? From the standpoint of the present model, it might well have more to do with physical laws rather than with the information theoretic structure of Hilbert space. However, interestingly, such a probabilistic limit on accessible information has been argued as indicative of the informational nature of quantum mechanics [58, 89].

Is nature a computer? It is certainly not a digital computer running Linux or Windows. That information processing and computations actually underlie, rather than merely offer an interprettation of, the existence and evolution of physical reality is a controversial yet rich scientific paradigm that has been explored to various degrees in other contexts [57, 58, 59, 89]. In our model, we find that the classicality of measurement outcomes and of usual macroscopic systems reflects an underlying information processing threshold, related to the finite fine-graining of Hilbert space, which is highly suggestive of an information theoretic origin of physical reality. In this viewpoint, nature can arguably be thought of as an abstract operating system running a fundamental unitary program, that is continually interrupted by entanglement-engendered memory-overflows at macroscopic scales.

The dynamics of the information transitions depends on the computational architecture of this 'operating system' underlying physical systems, that may not appear in the arena of physical dynamics but instead at the deeper subquantum level. Here it is of interest to study whether the free parameter of the computational model, namely $\mu$, 
emerges as a basic information processing feature of nature, that can be linked in intresting ways to other aspects of physics, in particular quantum gravity and quantum cosmology. The computational model complements decoherence in quantum measurements, by turning the improper mixture of decoherentially localized particles of the subsystem of interest into a proper mixture. It thereby helps complete the measurement act and offers a resolution to the measurement problem. It does so adding arguably minimal new physics in almost all experimentally accessible regimes.

The model implies that classicality at the macroscopic level arises because ever-increasing entanglement can 'overwhelm' nature's computational capacity. In view of Eq. (17), it roughly reflects the fact that $\mu \ll N_{p} \approx 10^{120}$, where $N_{p}$ is the number of degrees of freedom in the universe. In a universe where $\mu \gg N_{p}$, quantum mechanics would possess enough mnemonic and computational resource to support the entangled evolution of all matter and energy. Information transitions, and hence the classicality of measurement outcomes and macroscopic matter, would be absent. Macroscopic phenomena will regularly be quantum mechanical. Decoherence will still causes localization of particles, but superpositions of such decohered/localized particles will persist. So, why is $\mu$ relatively so small? The question can be paraphrased conversely: why is the universe relatively so large? This is analogous to a situation in astronomy: that the large scale evolution of the universe is slow can be viewed as due to the relative slowness of light's speed, or as due to the immense size of the universe. One can also ask if there is some sort of Dirac-like large number hypothesis [38] that can connect $\mu$ and $N_{p}$.

The idea of states of physical systems and their evolution deriving from an information processing substratum raises deep philosophical questions: What is the basic hardware on which the 'operating system' called nature is mounted? Can one go farther and, as suggested in Section V regard physical objects themselves as information theoretic constructs? What sort of tests would be needed to elevate such a hypothesis beyond the status of a mere interpretation to an ontology (just as macroscopic classicality is said to indicate the computational nature of physical evolution of systems)? And what does this imply for and how does it relate to quantum gravity, a program that, inspite of the recent spectacular successes in string and $\mathrm{M}$ theories [55] and loop quantum gravity [56], proves to be a very difficult and elusive goal? It is hoped that further developments in the relation between quantum physics and computation will shed light on these, and related, questions.

What are there decisive tests of the model? The very classicality of measurement outcomes and macroscopic matter is, in our opinion, a relevant evidence, in view of the Bassi-Ghirardi theorem [8]. However, the evidence is not clinching, because collapse theories and Many-worlds interpretations also predict the same. Other than this, evidence from mesoscopic systems, specifically experiments of the types described in Refs. [69, 70], will be important. This is because the predictions of the computational model are indistinguishable from quantum mechanics for microscopic systems and open macroscopic systems. Now, like collapse models, the computational model implies that even closed macroscopic systems exist in mixed state and show irreversible behaviour. In practice, it is rather difficult to test this, because there are rarely truely isolated macroscopic systems, other than condensates and superfluids, which, as noted earlier, present qualitatively different situations. Even interstellar dust grains are constantly scattering cosmic background radiation and are thus open [17].

To be able to resolve the matter, careful study of isolated, mesoscopic systems is needed. This is no easy task experimentally, not only from the viewpoint of noise-control, but also because the information transitional effects would have to be distinguished from quantum chaotic and decoherence effects. But such studies alone can shed more light on the onset of decoherence and the emergence of computational instability and also enable us to fix $\mu$, the free parameter for the computational model, and thence $\mathcal{M}_{2}$ and $\lambda_{u}$.

I am thankful to Messrs. Kaushik Mitra and Sudhir Rao for discussions and pointing out important references. I thank Prof. J. Pasupathy for useful discussions. This work, begun at the Center for Theoretical Studies, Indian Institute of Science, was partially supported by the DRDO510 project no. 01PS:00356.

[1] J. Krips, "Measurement in Quantum Theory", The Stanford Encyclopedia of Philosophy, Rob Clifton (ed.), $\mathrm{URL}=$ http://plato.stanford.edu/entries/qt-measurement/

[2] J. A. Wheeler and W. H. Zurek, Quantum Theory and Measurement (Princeton University Press, 1983).

[3] G. Ghirardi, Phys. Lett. A 262, 1 (1999).

[4] D. Home, Conceptual Foundations of Quantum Mechanics: An overview from Modern Perspectives (Plenum, New York 1997).

[5] M. A. Nielsen and I. Chuang, Quantum Computation and Quantum Information, (Cambridge 2000).

[6] J. Preskill, Lecture Notes on Quantum Computation, http://www.theory.caltech.edu/people/preskill/p299/\#lecture

[7] J. von Neumann, The Mathematical Foundations of Quantum Mechanics, translated from German by R. T. Beyer (Princeton Univ. Press 1955).

[8] A. Bassi and G. Ghirardi, Phys. Lett. A 275, 373 (2000). 
[9] E. Schrödinger, Naturwissenschaften 23, 807; 823; 844. (1935). English translation: John D. Trimmer, Proceedings of the American Philosophical Society, 124, 323-38 (1980), Reprinted in Quantum Theory and Measurement, pp. 152 (1983).

[10] E. Joos and H. D. Zeh, Z. Phys. B59, 223, 1985.

[11] W. H. Zurek, Phys. Rev. D24, 1516, 1981; D26, 1862, 1982.

[12] W. H. Zurek, Phys. Today, October 1991, p. 36.

[13] H. D. Zeh, in Proc. of Bielefeld conference on Decoherence: Theoretical, Experimental and Conceptual Problems, eds. P. Blanchard, B. Giulini, R. Joos, C. Kiefer, I.- O. Stamatescu (Springer 1999); eprint quant-ph/9905004

[14] A. Peres, Phys. Rev. A 61022116 (2000).

[15] D. A. Kokorowski, A. D. Kronin, T. D. Roberts and D. E. Pritchard, Phys. Rev. Lett. 86, 2191 (2001).

[16] S. L. Adler, eprint quant-ph/0112095.

[17] R. Joos, in Proc. of Bielefeld conference on Decoherence: Theoretical, Experimental and Conceptual Problems, eds. P. Blanchard, B. Giulini, R. Joos, C. Kiefer, I.- O. Stamatescu (Springer 1999) p. 1-17.

[18] B. d'Espagnat, in Preludes in Theoretical Physics, ed. A. De-Shalit, A. Feshbach and L. van Hove (1966, North Holland).

[19] A. Peres, Phys. Rev. A61, 022116.

[20] For example, a selective measurement in Ref. 31] apparently involves an abrupt 'jump' from an unrestricted set of worldlines in the Feynman propagator integral to narrower corridor of worldlines, consistent with the measurement read-out.

[21] H. Everett III, Rev. Mod. Phys. 29, 454, 1957.

[22] B. S. DeWitt, The Many-universes Interpretation of Quantum Mechanics in Foundations of Quantum Mechanics, (Academic Press, New York, 1971); reprinted in B. S. DeWitt and N. Graham (eds): p. 167 The Many-Worlds Interpretation of Quantum Mechanics, (Princeton University Press, 1973).

[23] J. Barrett, "Everett's Relative-State Formulation of Quantum Mechanics", The Stanford Encyclopedia of Philosophy (Fall 2002 Edition), Edward N. Zalta (ed.), URL = http://plato.stanford.edu/archives/fall2002/entries/qm-everett/

[24] For example, if the initial state of $S$ in Eq. (11) is $(1 / \sqrt{2})((1 / \sqrt{23}|0\rangle+\sqrt{22 / 23}|1\rangle)$ in the measurement basis, it would suggest that a measurement in the $\{|0\rangle,|1\rangle\}$ basis, in order to conform to the Born probability law, must cause the universe to branch out into 23 versions, exactly 1 of which goes into the $|0\rangle$ state. And if the coefficients $a_{i}$ are irrational, then each measurement would seem to cause the universe to branch out into infinite versions.

[25] D. Albert and B. Loewer: Interpreting the Many Worlds Interpretation, Synthese 77, 195 (1988).

[26] R. B. Griffiths, J. Stat. Phys. 36, 219 (1984).

[27] R. Omnès, J. Stat. Phys. 53, 893 (1988).

[28] M. Gell-Mann and J. B. Hartle: in Complexity, Entropy, and the Physics of Information, Proc. of the Santa Fe Inst. Studies in the Sciences of Complexity vol. VIII, ed. W. H. Zurek (Addison-Wesley, 1996).

[29] N. D. Mermin, Pramana 51549 (1998); (eprint quant-ph/9609013); S. Saunders, Synthèse 102, 235 (1995).

[30] J. Barrett, Erkenntnis 41233 (1994); J. Barrett, The Quantum Mechanics of Minds and Worlds, (Oxford University Press, 1998); F. A. Dowker and A. Kent: eprint gr-qc/9412067

[31] M. Mensky, eprint quant-ph/0212112

[32] M. Samal and P. Ghosh, eprint quant-ph/0202176

[33] D. Bohm, The Undivided universe (Routledge, 1993).

[34] G. Ghirardi, A. Rimini and T. Weber, Phys. Rev. D 34470 (1986); G. C. Ghirardi, R. Grassi, F. Benatti, Found. of Phys., 25, 5 (1995). G. C. Ghirardi, P. Pearle, A. Rimini Phys. Rev. A 42, 78 (1990).

[35] G. Ghirardi, "Collapse Theories", The Stanford Encyclopedia of Philosophy (Fall 2002 Edition), Edward N. Zalta (ed.), $\mathrm{URL}=$ http://plato.stanford.edu/archives/fall2002/entries/qm-collapse/

[36] R. Penrose, Gen. Rel. Grav. 28, 581 (1996).

[37] S. Lloyd, Nature 407, 1047 (2000).

[38] S. Lloyd, Phys. Rev. Lett. 88 (2002) 237901; eprint quant-ph/0110141

[39] C. Sivaram, Current Science 79, 413 (2000).

[40] Y. J. Ng, Phys. Rev. Lett. 862946 (2001); Phys. Rev. Lett. 88139902 (2002).

[41] A. K. Pati, S. R. Jain, A. Mitra and R. Ramanna, Phys. Lett. A 301, 125 (2002).

[42] N. Margolus and L. B. Levitin, in PhysComp96, Proceedings of the Fourth Workshop on Physics and Computation, eds. T. Toffoli, M. Biafore and J. Leão (New England Complex Systems Institute, Boston 1996); Physics (Amsterdam) 120D, 188 (1998).

[43] R. Landauer, Nature (London) 335, 779 (1988).

[44] J. D. Bekenstein, Phys. Rev. D23, 287 (1981); Phys. Rev. Lett. 46, 623 (1981); Phys. Rev. D30 1669 (1984).

[45] An Introduction to Kolmogorov Complexity and Its Applications, Ming Li and Paul Vitanyi, (Springer Verlag 1997).

[46] W. K. Wootters, Phys. Rev. D 23, 357 (1981);

[47] S. L. Braunstein and C. M. Caves, Phys. Rev. Lett. 72, 3439 (1994).

[48] R. Schack, G. M. D'Ariano and C. M. Caves, Phys. Rev. E 50, 972 (1994).

[49] J. von Neumann, Theory of Self-reproducing Automata Lect. 3 (Univ. of Illinois, Urbana, 1966).

[50] W. K. Wooters and W. H. Zurek, Nature 299, 802 (1982).

[51] According to the holographic principle [40], the maximum information that can be registered in a system is $A / l_{P}^{2}$, where $A$ is the system's area. This would suggest $\mathcal{M}=\mu\left(V / V_{P}\right)^{2 / 3}$. This may be relevant in the quantum gravity regime, but for our purposes, we will quanitfy $\mathcal{M}$ as the volume Kolmogorov-Chaitin complexity.

[52] A. Einstein, B. Rosen and B. Podolsky, Phys. Rev. 47, 777 (1935).

[53] P. Shor, SIAM Journal of Computing 26, 1484 (1997). 
[54] R.P. Feynman, Int. J. Theor. Phys. 21, 467, 1982.

[55] T. Banks, W. Fischler, S. H. Shenker, and L. Susskind, Phys. Rev. D 55, 5112 (1997).

[56] Lee Smolin, Three Roads to Quantum Gravity (London: Weidenfeld and Nicholson, 2000); L. Crane, A. Perez and C. Rovelli, Phys. Rev. Lett. 87, 181301 (2001).

[57] R. T. Cahill and C. M. Klinger, Phys. Lett. A223, 313 (1996); R. T. Cahill and C. M. Klinger, Gen. Rel. \& Grav. 32, 529 (2000); R. T. Cahill, C. M. Klinger and K. Kitto, The Physicist, 37, 191 (2000).

[58] A. Zeilinger, Found. of Phys., 29, 631 (1999).

[59] S. Wolfram, A New Kind of Science (Wolfram Media, 2002).

[60] H. Pagels, The Cosmic Code: Quantum Physics as the Language of Nature (Simon and Schuster, New York 1982).

[61] A. Patel, Jl. of Biosciences 27, 207 (2002).

[62] B. Georgeot and D. L. Shepelyansky, Phys. Rev. E 623504 (2000); B. Georgeot and D. L. Shepelyansky, Phys. Rev. E 62, 6366 (2000).

[63] F. J. MacWilliams and N. J. A. Sloane, The Theory of Error-correcting Codes (North-Holland 1977).

[64] Actually, the required clock rate should be $\omega_{\chi}\left(\mathcal{D}^{\prime} \mathcal{P} / \mathcal{M}\right)$ where $x=((1 / r)-1)$ and $\mathcal{P}$ is the number of elements in the parity check matrix [63], given by $\mathcal{P}=\mu^{2} n^{2} / r$. For our estimate we ignore $\mathcal{P}$, which is polynomial in $n$. Also note that we are invoking a simple classical error correction procedure, rather than quantum error correction [68], as the correction is supposed to be implemented on the state information rather than on quantum states.

[65] Segmentation fault occurs when there is invalid pointer de-referencing. In our case, the pointer is invalid because it is trying to access a piece of unallocated (or de-allocated) memory. In conventional computing, it can be invalid also because it is a NULL-pointer or an un-initialized pointer.

[66] A computer program is said to crash when it suffers a sudden major failure usually with attendant loss of data (MerriamWebster dictionary; http://www.m-w.com).

[67] The core is the file that contains a snapshot of the memory registers at the time when a program crashes. It is 'dumped' into a compiler-specified directory when a program crashes.

[68] Calderbank and P. Shor, Phys. Rev. A 54, 1098 (1996); A. Steane, Proc. Roy. Soc., London, Ser. A 452, 2551 (1996).

[69] M. Arndt, O. Nairz, J. Vos Andrae, C. Keller, G. van der Zouw and A. Zeilinger, Nature 401, 680 (1999).

[70] E. Altewischer, M. P. van Exter and J. P. Woerdman, Nature 418, 304 (2002).

[71] The completeness of the measurement operators $E_{i}$ is sufficient to prove this, ie., $\sum_{i} E_{i}=\mathbb{I}$. If such completeness is not guaranteed, nonlocal signaling would be possible (G. C. Ghirardi, A. Rimini, T. Weber, Lett. Nuovo Cimento, 27, 293 (1980); R. Srikanth, Pramana 59, 169 (2002); errata in ibid. 59, 1076 (2002)). However, this still does not affect the 'derivation' of the Born rule for projective measurements in the computational model.

[72] S. Mayburov, eprint quant-ph/0212099

[73] D. Bruss, eprint quant-ph/0110078

[74] C. J. Myatt, B. E. King, Q. A. Turchette, C. Sackett, D. Kielpinski, W. Itano, C. Monroe, \& D. J. Wineland, Nature 403, 269 (2000).

[75] A. Patel, Journal of Genetics 8039 (2001).

[76] In fact, computational stability is only necessary but not sufficient to manifest coherent wavelike behaviour. In practice, it will also be necessary to take into consideration various energy or wavelength scales of the system (rotational, vibrational, etc.). The presence of such multiple internal degrees of freedom of comparable energy can lead to broad-band wavelength scales that can potentially wash out interference patterns.

[77] W. Marshall, Christoph Simon, R. Penrose, D. Bouwmeester, eprint quant-ph/0210001

[78] S. Yu. Kun, L. Benet, L. T. Chadderton, W. Greiner, F. Haas, Phys. Rev. C, 67, 011604R (2003).

[79] E. F. Wigner, Rev. Mod. Phys. 25, 255 (1957).

[80] V. Scarani, W. Tittel, H. Zbinden, N. Gisin, Phys. Lett. A 2761 (2000).

[81] J. S. Bell, Physics 1, 195 (1964).

[82] R. Garisto, eprint quant-ph/0212078

[83] H. Zbinden, J. Brendel, N. Gisin and W. Tittel, Phys. Rev. A 63, 022111-1 (2001).

[84] J. Rembielinski and K. A. Smolinski, Phys. Rev. A66, 052114 (2002).

[85] G. Lindblad, Commun. Math. Phys. 48, 119 (1976).

[86] V. Gorini, A. Kossakowski and E. C. G. Sudarshan, J. Math. Phys. 17, 821 (1976).

[87] J. Anglin and W. Ketterle, Nature 416211 (2002).

[88] J. R. Friedman, V. Patel, W. Chen, S. K. Tolpygo, J. E. Lukens, Nature 406, 43 (2000).

[89] M. Mehrafarin, Found. Phys. Lett. 16 (2003) 127 (eprint quant-ph/0205028). 